\title{
19. MINERALOGY OF SEDIMENTS FROM THE MIDDLE AMERICA TRENCH (GUATEMALA TRANSECT) ${ }^{1}$
}

\author{
Victor Kurnosov, Far-East Geological Institute, U.S.S.R. Academy of Sciences, Vladivostok, U.S.S.R. \\ and
}

Ivar Murdmaa, Vera Kazakova, Vladlena Mikhina, and Alla Shevchenko, Shirshov Institute of Oceanology, U.S.S.R. Academy of Sciences, Moscow, U.S.S.R.

\section{INTRODUCTION}

Seven sites were drilled during Leg 67 along a transect across the Middle America Trench off Guatemala: four (Sites 494, 496, 497, and 498) on continental slope, two (Sites 499 and 500) on Trench floor, and one (Site 495) on the Cocos Plate. We studied the mineralogy of sediments from Sites 494, 495, 496, 499, and 500. Our objective was to investigate the origin and source of separate minerals and mineral assemblages, giving special attention to the influence of the alteration of basalts on the sediment mineralogy, which we expected to be particularly important in layers just above oceanic basement.

\section{METHOD}

We analyzed our samples using the following methods: general description of smear-slides and thin sections for the entire sample, immersion method for the coarse silt fraction $(0.1-0.05 \mathrm{~mm})$, X-ray diffractometry for the quantitative determination of clay minerals (using the Biscaye method, Biscaye, 1964) and for identification of other major minerals in the size fractions less than $2 \mu \mathrm{m}$ and 2 to $20 \mu \mathrm{m}$; and electron microscopy for the study of clay mineral microstructures. Electron diffraction and infrared spectroscopy were utilized as additional diagnostic methods.

\section{MINERALS}

Clay minerals. The clay minerals constitute an essential part of the Neogene to Quaternary terrigenous mud and occur as a minor admixture in calcareous sediments. In all samples, smectite predominates over other clay minerals. Mica, chlorite, and kaolinite occur in all samples in minor quantities, and mixed-layer chlorite-montmorillonite is rare. Other minerals identified by X-ray in the fine fractions of the terrigeneous and diatomaceous mud are feldspar, quartz, clinoptilolite, and amorphous phase (i.e., of unknown composition). In the calcareous sediments, calcite content is so high that quantitative determination of clay minerals is impossible.

Smectite from the noncalcareous sediments is a dioctahedral ferric montmorillonite (parameter $b=9.02$ $9.06 \AA$ ), soluble in hot $10 \% \mathrm{HCl}$. It probably contains interlayered mica sheets, which were detected by infrared spectroscopy, as well as by $Z$ coefficient (relation between two shoulders of the 17 - $\AA$ reflection peak), averaging 0.5 . The smectite particles are isometric flakes

\footnotetext{
${ }^{1}$ Aubouin, J., von Huene, R., et al., Init. Repts. DSDP, 67: Washington (U.S. Govt. Printing Office).
}

of cloudy appearance (Plate 1, Fig. 1), similar throughout the sediment.

Smectite in calcareous sediments is also a dioctahedral $\mathrm{Fe}$-montmorillonite soluble in $10 \% \mathrm{HCl}(b=9.05-$ $9.06 \AA)$, but morphotogically it differs from the smectite in noncalcareous sediments in that its crystals are needleor ribbon-shaped (Plate 1, Figs. 2, 3, and 4).

Kaolinite commonly shows a wide low reflection about $7.13 \AA$ to $7.25 \AA$. It was rarely observed on the electron photomicrographs as pseudohexagonal particles (Plate 1, Fig. 1). The presence of kaolinite was confirmed by infrared spectra. Reflection 002 of kaolinite is well recognizable after chlorite dissolution in $10 \% \mathrm{HCl}$. The wideness of kaolinite reflection 001 is indicative of its imperfect structure. Kaolinite was not detected in calcareous sediments.

Chlorite, mixed-layer chlorite-montmorillonite, and even rarer mica are hardly detectable because of their scarcity. A minor amount of mica was found in a sample of calcareous sediment from Hole 499B-Sample 499B-9, CC (25-27 cm) - after dissolution of smectite in $10 \% \mathrm{HCl}$.

Clastic minerals. We detected clastic terrigeneous minerals both by X-ray for the size fractions $<2 \mu \mathrm{m}$ and 2 to $20 \mu \mathrm{m}$ and by optic microscopy of thin sections for the size fraction 0.1 to $0.05 \mathrm{~mm}$. Quartz we identified using reflections $4.23 \AA$ and $3.34 \AA$, and feldspar using reflections $3.20 \AA$ and $3.18 \AA$, the most prominant ones. Feldspars are common or abundant in most samples of noncalcareous sediments and commonly predominate over quartz. As indicated by their refraction indexes, the feldspars are represented mainly by calcic or intermediate plagioclase (andesine to labradorite) with minor amounts of albite-oligoclase and potassium feldspar.

Other clastic minerals found in the coarse silt fraction and in thin sections are: mica, chlorite, serpentine, acidic volcanic glass, basic volcanic glass, and palagonite (in light fraction); clinopyroxene, orthopyroxene, hornblende, actinolite-tremolite, epidote, olivine, garnet, zircon, sphene, anatase, apatite, corundum, biotite, chlorite, spinel, and black opaques (mainly magnetite)-in heavy fraction (Table 1).

Authigenic minerals. By X-ray we detected in noncalcareous sediments authigenic clinoptilolite (using the poorly developed reflection $8.9 \AA$ ) and trace amounts of cristobalite or opal-CT (using reflections 4.04-4.06 $\AA$ ). In thin sections, smear slides, and the coarse silt fraction 
Table 1. Coarse silt (0.1-0.05 mm) fraction mineralogy as determined by immersion method (percentage of grains counted). Part 1 . Heavy fraction. Part 2. Light fraction.

\begin{tabular}{|c|c|c|c|c|c|c|c|c|c|c|c|c|c|c|c|}
\hline $\begin{array}{c}\text { Sample } \\
\text { (core-section, } \\
\text { interval in cm) }\end{array}$ & $\begin{array}{c}\text { Sub-bottom } \\
\text { Depth (m) }\end{array}$ & $\begin{array}{l}\text { Transparent Minetals in } \\
\text { the Heavy Fraction }(\%)\end{array}$ & Garnet & Hornblende & Actinolite & Orthopyroxene & Clinopyroxene & Epidote & Olivine & Biotite & Barite & Apatite & Spinel & Zircon & Sphene \\
\hline \multicolumn{16}{|l|}{ Part 1. Heavy Fraction } \\
\hline \multicolumn{16}{|l|}{ Hole 494} \\
\hline $\begin{array}{l}1-1,33-38 \\
2-3,32-57\end{array}$ & $\begin{array}{r}0.3 \\
12.5\end{array}$ & $\begin{array}{l}45 \\
68\end{array}$ & $\overline{-}$ & $\begin{array}{l}14 \\
22\end{array}$ & $\bar{z}$ & $\begin{array}{l}13 \\
27\end{array}$ & $\begin{array}{l}70 \\
48\end{array}$ & ${ }_{1}^{2}$ & $\bar{z}$ & $\overline{-}$ & $\overline{-}$ & $\bar{z}$ & $\overline{-}$ & $\bar{z}$ & $\bar{z}$ \\
\hline $4-3,40-44$ & 31.4 & 77 & + & 15 & $=$ & 37 & 45 & $\mathrm{i}$ & - & - & - & - & - & 1 & - \\
\hline \multicolumn{16}{|l|}{ Hole 494A } \\
\hline $2-6,88-93$ & 55.9 & 27 & - & 8 & - & 24 & 61 & 1 & - & 1 & 2 & 1 & - & 2 & - \\
\hline $5-1,20-30$ & 75.8 & 47 & + & 8 & - & 35 & 54 & 1 & - & + & - & - & - & - & - \\
\hline $9-1,120-125$ & 114.7 & 45 & - & 11 & - & 32 & 55 & - & - & - & 1 & - & 1 & $\overline{2}$ & $\bar{z}+x+3$ \\
\hline $17-1,133-137$ & 190.9 & 51 & + & 25 & 1 & 20 & 49 & - & - & + & + & 1 & - & 2 & - \\
\hline \multicolumn{16}{|l|}{ Hole 495} \\
\hline $3-1,42-47$ & 19.4 & 13 & 6 & 41 & - & 6 & 46 & - & - & - & - & - & - & - & - \\
\hline $4-1,44-48$ & 28.9 & 12 & - & s & - & 7 & 85 & 3 & - & - & - & - & - & - & - \\
\hline $4.5,110-114$ & 35.6 & 25 & - & 50 & - & 8 & 36 & 4 & - & - & - & 1 & - & - & - \\
\hline $6-4,16-20$ & 52.2 & 30 & - & 56 & - & 12 & 30 & 1 & - & - & $\overline{-}$ & - & - & - & z \\
\hline $7-1,26-30$ & 57.3 & 18 & 4 & 12 & - & 28 & 48 & 4 & 4 & - & - & - & - & - & - \\
\hline $9-2,20-25$ & 77.2 & 9 & - & - & - & 8 & so & 4 & - & - & 38 & - & - & - & - \\
\hline $\begin{array}{l}12-5,21-26 \\
13.3,36-58\end{array}$ & 110.7 & 12 & - & $\overline{20}$ & 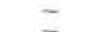 & 17 & 83 & - & - & $=$ & $\bar{z}$ & - & - & $\bar{z}$ & - \\
\hline $\begin{array}{l}13 .-3,36-58 \\
15.2,70-72\end{array}$ & $\begin{array}{l}1177.5 \\
135.2\end{array}$ & 35 & $=$ & 29 & $=$ & 29 & $\begin{array}{l}43 \\
95\end{array}$ & $=$ & $\bar{z}$ & $=$ & $\bar{z}$ & $\overline{-}$ & $\bar{z}$ & $\bar{z}$ & $=$ \\
\hline $16-4,50-54$ & 133.2 & $\begin{array}{l}37 \\
36\end{array}$ & $=$ & $\overline{3}$ & $\Xi$ & $-\frac{3}{-}$ & $\begin{array}{l}95 \\
81\end{array}$ & $\overline{12}$ & $\overline{-}$ & $\overline{-}$ & $\overline{5}$ & $\bar{z}$ & $\overline{-}$ & $=$ & $\bar{z}+$ \\
\hline $17-6,50-54$ & & 37 & - & - & - & 15 & 85 & - & - & - & - & - & - & - & - \\
\hline 18-5, 70-74 & 168.2 & 90 & - & 10 & - & 24 & - & 1 & - & 65 & - & - & - & - & - \\
\hline 19-4, $120-124$ & 176.7 & 41 & - & - & - & 62 & 34 & $\overline{20}$ & $=$ & $=$ & $\bar{z}+$ & 3 & $\bar{z}+$ & $=$ & $=$ \\
\hline $\begin{array}{l}20-5,138-142 \\
21-4,40-45\end{array}$ & $\begin{array}{l}187.9 \\
194.9\end{array}$ & ${ }_{29}^{43}$ & $\bar{z}$ & -18 & - & - & 80 & 20 & - & $\bar{z}$ & $\bar{z}$ & $=$ & $\bar{z}$ & $\bar{\pi}$ & $\bar{z}$ \\
\hline $22-1,20-25$ & 194.9 & $\begin{array}{l}29 \\
70\end{array}$ & $=$ & 18 & $=$ & $\overline{8}$ & $\begin{array}{l}82 \\
64\end{array}$ & $\overline{26}$ & $\bar{z}$ & $=$ & $\bar{z}$ & $\overline{2}$ & $=$ & $=$ & $\bar{z}$ \\
\hline $24-4,40-42$ & 223.0 & 6 & - & 14 & & 30 & 30 & 14 & - & - & - & -7 & - & - & - \\
\hline $26-1,40-44$ & & ? & 6 & 11 & - & - & 66 & - & - & - & - & 17 & - & - & - \\
\hline $32-1,35-39$ & 294.8 & 21 & 9 & 36 & - & 9 & 46 & - & - & - & - & - & - & - & - \\
\hline \multicolumn{16}{|l|}{ Hole 496} \\
\hline $3-1,50-54$ & 22.3 & 23 & - & 9 & - & 54 & 38 & - & - & - & - & - & - & - & - \\
\hline 6. & 50.9 & 41 & 2 & 8 & - & 50 & 41 & - & - & - & - & - & - & $\bar{z}+$ & $\bar{z}+$ \\
\hline 119 & 67.1 & 32 & - & 10 & - & 39 & 51 & - & - & - & - & - & - & - & - \\
\hline $\begin{array}{l}19-2,137-139 \\
224,2-33\end{array}$ & 171.9 & 17 & - & 24 & - & 32 & 44 & - & $\bar{z}$ & - & - & - & - & - & - \\
\hline $\begin{array}{l}22-4,28-33 \\
26-4,22-27\end{array}$ & $\begin{array}{l}202.3 \\
240.2\end{array}$ & ${ }_{74}^{8}$ & $=$ & 38 & z & 41 & 62 & $\overline{-}$ & $\bar{z}$ & $\bar{z}$ & $\bar{z}$ & $\bar{z}$ r & $\bar{z}$ & $\bar{z}$ & $\bar{z}$ \\
\hline $\begin{array}{l}26-4,22-27 \\
28-3,12-17\end{array}$ & $\begin{array}{l}240.2 \\
257.6\end{array}$ & $\begin{array}{l}74 \\
83\end{array}$ & $=$ & 至1 & $=$ & ${ }_{43}^{41}$ & $\begin{array}{l}48 \\
47\end{array}$ & $\bar{z}+$ & $=$ & $\bar{z}$ & $=$ & $\overline{-}$ & $\overline{-}$ & $\overline{-}$ & $\bar{z}$ \\
\hline $\begin{array}{l}28-3.6,58-62 \\
306\end{array}$ & $\begin{array}{l}257.6 \\
281.6\end{array}$ & $\begin{array}{l}83 \\
16\end{array}$ & $=$ & $\begin{array}{l}10 \\
33\end{array}$ & $=$ & $\begin{array}{l}43 \\
37\end{array}$ & ${ }_{31}^{47}$ & $\bar{z}+$ & $=$ & $=$ & $\overline{-}$ & $=$ & $\overline{-}$ & $\bar{z}+$ & $=$ \\
\hline $31-1,70-75$ & $\begin{array}{l}283.7 \\
283.7\end{array}$ & $\begin{array}{l}10 \\
36\end{array}$ & $=$ & $\begin{array}{l}33 \\
40\end{array}$ & $=$ & 39 & 14 & 7 & $=$ & $\overline{-}$ & - & - & $=$ & - & - \\
\hline \multicolumn{16}{|l|}{ Hole 499} \\
\hline I-1., $142-146$ & 1.4 & 57 & - & 35 & - & 33 & 29 & 2 & 2 & - & - & - & - & - & - \\
\hline $3 \cdot 2,80-8$ & 12.8 & 14 & - & 15 & - & 24 & 56 & 3 & - & - & - & - & - & - & - \\
\hline $5-2,10$ & 31.1 & 34 & - & 20 & - & 22 & 55 & 2 & 2 & - & - & - & - & - & - \\
\hline $6-$ & 43.5 & 81 & - & 19 & + & 27 & 51 & 2 & 1 & 1 & $=$ & - & - & - & z \\
\hline & 68.3 & 69 & - & 19 & - & 33 & 42 & 3 & 3 & - & - & - & - & 1 & - \\
\hline $\begin{array}{l}10-4,33-3 \\
12,38-6\end{array}$ & 81.8 & 80 & - & 10 & - & 28 & 58 & i & 3 & \pm & $\bar{z}+$ & - & $\bar{z}+$ & $\bar{z}+x$ & $\bar{z}$ \\
\hline $\begin{array}{l}12-3,58-63 \\
14-2,114-119\end{array}$ & $\begin{array}{r}99.6 \\
1116\end{array}$ & 年8 & $=$ & ${ }_{13}^{6}$ & $\overline{1}$ & $\begin{array}{l}211 \\
47\end{array}$ & $\begin{array}{l}69 \\
39\end{array}$ & 1 & -3 & $\overline{-}$ & $\bar{z}$ & \pm & $\bar{z}$ & $\bar{z}$ & $\overline{1}$ \\
\hline $\begin{array}{l}14-2,114-119 \\
15-2,46-48\end{array}$ & $\begin{array}{l}l_{126.5} \\
\text { 12.6. }\end{array}$ & $\begin{array}{l}61 \\
44\end{array}$ & $=$ & ${ }_{11}^{13}$ & $\frac{1}{-1}$ & $\begin{array}{c}47 \\
51\end{array}$ & $\begin{array}{l}39 \\
37\end{array}$ & $\overline{1}$ & $\overline{1}$ & $\overline{-}+x+$ & 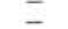 & $\overline{-}$ & $=$ & $=$ & 1 \\
\hline $18-4,80-84$ & $\begin{array}{l}158.3 \\
150.3\end{array}$ & 33 & $\overline{-}$ & 49 & $\Xi$ & ${ }_{21}^{11}$ & 22 & 3 & $\mathrm{i}$ & 1 & - & 2 & - & 1 & $=$ \\
\hline $\begin{array}{l}23-1,44-49 \\
25,49-98\end{array}$ & 200.9 & $\begin{array}{l}36 \\
78\end{array}$ & 5 & 36 & - & 14 & 27 & 9 & - & 9 & $\bar{z}$ & $\overline{7}$ & $\bar{z}$ & $\overline{-}$ & - \\
\hline $25-4,94-98$ & & 78 & 3 & 6 & & 6 & 14 & - & - & - & - & 71 & & - & \\
\hline
\end{tabular}

we observed authigenic pyrite in the form of globular aggregates, framboids, cubic crystals, and replacement of diatoms and radiolarians. In some intervals pyrite is quite abundant. Along with pyrite we found poorly developed glauconite, gypsum, barite, clinoptilolite, iron oxides, and manganese micronodules(?). The latter two occur in calcareous pelagic sediments.

Biogenic minerals. The biogenic mineral constituents of the sediments are represented by opal-A of diatom frustules, radiolarian skeletons, and sponge spicules (in hemipelagic mud), and by low-magnesium calcite of nannofossils and foraminifer tests. In the coarse silt fraction we also found biogenic phosphatic grains, likely bone debris. Plant debris and particles of organic matter are common in most samples of hemipelagic mud.

\section{MINERALOGY OF LITHOLOGIC UNITS}

We classified our mineralogical data by the lithologic units, which were distinguished by shipboard scientists for each hole. Our data cover most lithologic units, although some of these are characterized only by one or two samples.

\section{Site 494}

Site 494 is located on the lower continental slope of the Middle America Trench $3 \mathrm{~km}$ landward of the Trench axis at a water depth of 5472 meters. Holes 494 and $494 \mathrm{~A}$ penetrated 323 meters of sediments from Holocene to Upper Cretaceous underlain by magmatic rocks. The sedimentary section was subdivided into seven lithologic units, which are described in the site report (this volume). Our smear-slide descriptions are in a good accordance with these data, except for biogenic silica content, which, we suppose was somewhat overestimated by the shipboard sedimentologists. We estimated not more that $10 \%$ to $12 \%$ total biogenic silica in Unit 1 , which was designated "diatomaceous mud."

We studied samples downhole to a sub-bottom depth of 304 meters including 5 upper units (Table 2). X-ray data show rather uniform mineral composition of the units (Fig. 1). Smectite predominates throughout $(85 \%$ - 
Table 1. (Continued).

\begin{tabular}{|c|c|c|c|c|c|c|c|c|c|c|c|c|c|}
\hline \multirow{2}{*}{$\begin{array}{c}\text { Sample } \\
\text { (core-section, } \\
\text { interval in } \mathrm{cm} \text { ) }\end{array}$} & \multirow{2}{*}{$\begin{array}{l}\text { Sub-bottom } \\
\text { Depth }(m)\end{array}$} & \multirow[b]{2}{*}{ Quartz } & \multirow{2}{*}{$\begin{array}{l}\text { Feldspar } \\
(n<1.54)\end{array}$} & \multirow{2}{*}{$\begin{array}{l}\text { Plagioclase } \\
(n>1.54)\end{array}$} & \multirow[b]{2}{*}{ Chlorite } & \multirow[b]{2}{*}{ Glauconite } & Volcan & c Glass & & & & Bioger & \\
\hline & & & & & & & (acidic) & (basic) & Zeolite & Fragments $^{\mathbf{a}}$ & Calcite & Opal & Phosphate \\
\hline Part 2. Light fraction & & & & & & & & & & & & & \\
\hline Hole 494 & & & & & & & & & & & & & \\
\hline 1-1, 33-38 & 0.3 & 3 & - & 10 & 8 & - & 23 & 3 & 2 & 44 & 1 & 6 & - \\
\hline $\begin{array}{l}2-3,52-57 \\
4-3,40-44\end{array}$ & $\begin{array}{l}12.5 \\
31.4\end{array}$ & $\begin{array}{l}4 \\
2\end{array}$ & $\bar{z}$ & $\begin{array}{l}20 \\
30\end{array}$ & 5 & $\bar{z}$ & $\begin{array}{l}29 \\
34\end{array}$ & \pm & $\overline{2}$ & $\begin{array}{l}40 \\
25\end{array}$ & \pm & $\frac{2}{7}$ & $=$ \\
\hline Hole 494A & & & & & & & & & & & & & \\
\hline $2-6,88-93$ & 55.9 & 4 & - & 23 & 15 & - & 18 & + & 2 & 36 & - & 2 & - \\
\hline $5-1,26-30$ & 75.8 & - & 1 & 27 & 8 & - & 12 & - & & 47 & + & 2 & - \\
\hline $9-1,120-125$ & 114.7 & 2 & \pm & 21 & 4 & $\bar{z}+x+3$ & 29 & $\bar{z}+x \cdot+x$ & - & ${ }_{26}^{40}$ & $\bar{z}+x+1$ & 4 & $\bar{z}+1+x$ \\
\hline $\begin{array}{l}17-1,133-137 \\
20-5,46-50\end{array}$ & $\begin{array}{l}224.4 \\
224.4\end{array}$ & 9 & $\overline{1}$ & $\begin{array}{r}10 \\
9\end{array}$ & 6 & $=$ & 51 & $=$ & $\overline{1}$ & $\begin{array}{l}20 \\
16\end{array}$ & $=$ & 7 & - \\
\hline $22-2,14-21$ & 238.6 & $\underline{-}$ & 1 & 8 & $\underline{-}$ & $\overline{-}$ & is & $\bar{z}$ & - & so & $=$ & 25 & 2 \\
\hline $25-1,33-36$ & 265.8 & 1 & $=$ & $i$ & - & $\overline{-}$ & 13 & - & $=$ & 19 & 20 & 45 & - \\
\hline $27-1,39-44$ & 284.3 & 2 & - & 9 & + & - & 35 & - & - & 17 & 17 & 20 & - \\
\hline $29-1,11-12$ & 303.8 & $i$ & - & 17 & - & - & - & - & - & 3 & 69 & 10 & - \\
\hline Hole 495 & & & & & & & & & & & & & \\
\hline 3-1, $42-47$ & 19.4 & 2 & 2 & 12 & 1 & - & $\$ 4$ & - & - & 9 & 12 & 10 & + \\
\hline $4-1,44-48$ & 28.9 & - & $\div$ & is & i & - & 18 & - & 1 & 33 & $=$ & 31 & 1 \\
\hline $\begin{array}{l}4-5,110-114 \\
64,16-20\end{array}$ & $\begin{array}{l}55.6 \\
52.2\end{array}$ & 1 & 1 & $\begin{array}{r}6 \\
15\end{array}$ & $\overline{3}$ & $\overline{-}$ & $\begin{array}{l}75 \\
18\end{array}$ & $=$ & $\overline{1}$ & 4 & $=$ & ${ }_{31}^{14}$ & $\overline{1}$ \\
\hline $\begin{array}{l}6-4,1,26-20 \\
7-1,26-30\end{array}$ & $\begin{array}{l}32.2 \\
57.3\end{array}$ & $\mp$ & $\mp$ & $\begin{array}{l}15 \\
4\end{array}$ & - & $\bar{z}+$ & 衴 & $\overline{+}$ & - & $\begin{array}{r}31 \\
6\end{array}$ & $\overline{1}$ & $\begin{array}{l}31 \\
37\end{array}$ & 1 \\
\hline $\begin{array}{l}-1.1 .20-30 \\
9-2,20-25\end{array}$ & $\begin{array}{l}37.3 \\
77.2\end{array}$ & $\begin{array}{lll}+ \\
1\end{array}$ & $i_{i}^{+}$ & ${ }_{5}^{4}$ & $\overline{-}$ & $=$ & $\begin{array}{l}51 \\
44\end{array}$ & \pm & $=$ & $\begin{array}{l}6 \\
4\end{array}$ & 2 & 45 & $\overline{-}$ \\
\hline $12-5,21-26$ & 110.7 & 2 & 2 & 4 & $\overline{-}$ & $=$ & 67 & $\bar{z}$ & $\bar{z}$ & 5 & - & 21 & $\mp$ \\
\hline $13-3,56-58$ & 117.6 & + & - & 2 & 1 & $=$ & 81 & $\overline{-}$ & $\overline{-}$ & 1 & - & is & - \\
\hline $15-2,70-72$ & 135.2 & - & - & + & - & $=$ & 56 & + & - & 30 & - & 13 & - \\
\hline $16-4,50-54$ & & + & - & 2 & - & - & 51 & - & - & 13 & - & 31 & 3 \\
\hline $\begin{array}{l}18-5,70-74 \\
19-4,120-124\end{array}$ & $\begin{array}{l}168.2 \\
176.7\end{array}$ & 6 & - & 5 & - & $=$ & 50 & ${ }^{3}$ & 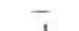 & ${ }^{4}$ & $=$ & 32 & $\overline{2}$ \\
\hline $\begin{array}{l}19-4,1020-124 \\
20-5,138-142\end{array}$ & $\begin{array}{l}187.9 \\
187.9\end{array}$ & 1 & $=$ & 6 & + & $=$ & $\begin{array}{l}73 \\
70\end{array}$ & \pm & 1 & ${ }_{2}^{4}$ & $\overline{40}$ & $\begin{array}{l}13 \\
21\end{array}$ & ${ }_{4}^{2}$ \\
\hline $21-4,40-45$ & 194.9 & + & $=$ & $=$ & 1 & $\bar{z}$ & 1 & $\overline{-}$ & $=$ & ${ }_{2}^{2}$ & $\begin{array}{l}40 \\
95\end{array}$ & + & ${ }_{1}^{4}$ \\
\hline $22-1,20-25$ & & - & - & - & - & - & 7 & 22 & - & 1 & 53 & 17 & $\frac{1}{2}$ \\
\hline $23-3,80-84$ & & 1 & - & - & - & - & 1 & - & - & i & 84 & 10 & ${ }_{3}^{2}$ \\
\hline $\begin{array}{l}24-4,40-42 \\
25-6,10-15\end{array}$ & 223.4 & 1 & - & 1 & - & - & - & - & - & 3 & $\ddot{*}$ & 95 & - \\
\hline $\begin{array}{l}25-6,10-15 \\
26-1,40-44\end{array}$ & 235.6 & - & - & 1 & - & - & 43 & - & - & 4 & $\ddot{*}$ & 52 & - \\
\hline $\begin{array}{l}26-1,40-44 \\
26-3,40-44\end{array}$ & & $\begin{array}{l}1 \\
+\end{array}$ & \pm & $=$ & $\bar{z}$ & $\bar{z}$ & ${ }_{1}^{3}$ & $\bar{z}$ & $\bar{z}$ & 3 & 48 & 41 & 2 \\
\hline $27-3,7-12$ & 250.1 & + & - & $=$ & $\overline{-}$ & $\overline{-}$ & 3 & $\overline{-}$ & $=$ & 1 & $\ddot{36}$ & 98 & - \\
\hline $28-7,5-9$ & 265.6 & - & - & - & 2 & - & - & & $=$ & $\overline{10}$ & $\begin{array}{l}36 \\
33\end{array}$ & 52 & ${ }_{3}^{4}$ \\
\hline $\begin{array}{l}28-2,30-35 \\
29-4,80-82\end{array}$ & $\begin{array}{l}258.3 \\
271.3\end{array}$ & $\bar{t}$ & $\bar{z}+$ & $=$ & $=$ & $=$ & - & - & 1 & 7 & 33 & 56 & 3 \\
\hline $\begin{array}{l}29-4,80-82 \\
30-2,20-24\end{array}$ & $\begin{array}{l}271,3 \\
277.2\end{array}$ & \pm & $\bar{z}$ & $\overline{-}$ & $\overline{2}$ & $\bar{z}$ & $\bar{z}$ & $\bar{z}$ & - & & 68 & & 3 \\
\hline $\begin{array}{l}30-2,20-24 \\
31-5,17-21\end{array}$ & $\begin{array}{l}227.2 \\
291.2\end{array}$ & $=$ & $=$ & $\overline{-}$ & 2 & $=$ & $\overline{2}$ & $=$ & $=$ & 2 & 26 & 70 & - \\
\hline $32-1,35-39$ & 294.8 & 1 & $=$ & + & $\bar{z}+$ & $=$ & i & $\bar{z}$ & $\bar{z}$ & 1 & $*$ & 97 & $\bar{z}$ \\
\hline $33-5,20-25$ & 310.2 & 2 & - & 1 & - & - & 3 & - & $=$ & 2 & $\ddot{*}$ & 92 & $=$ \\
\hline $\begin{array}{l}35-3,16-20 \\
37-3,51-53\end{array}$ & $\begin{array}{l}326.2 \\
345.5\end{array}$ & + & $=$ & - & - & $\bar{z}$ & - & - & + & - & 81 & 18 & - \\
\hline Hole 496 & 343.9 & + & - & - & - & - & 1 & - & & - & 97 & - & 2 \\
\hline Hole 496 & & & & & & & & & & & & & \\
\hline $\begin{array}{l}3 \cdot 1,50-54 \\
6-4,86-91\end{array}$ & 22.3 & - & - & 10 & - & - & 43 & - & - & 36 & 5 & 6 & - \\
\hline $\begin{array}{l}64,86-91 \\
84-2,14-119\end{array}$ & $\begin{array}{l}50.9 \\
67.1\end{array}$ & 1 & $=$ & 10 & 4 & $=$ & $\begin{array}{l}30 \\
23\end{array}$ & $\bar{z}+$ & $\bar{z}+$ & $\begin{array}{l}37 \\
43\end{array}$ & ${ }_{4}^{2}$ & $\begin{array}{l}16 \\
16\end{array}$ & + \\
\hline $19-2,137-139$ & $\begin{array}{l}67.1 \\
171.9\end{array}$ & $\overline{5}$ & $=$ & $\begin{array}{l}10 \\
10\end{array}$ & $\begin{array}{l}3 \\
2\end{array}$ & $\Xi$ & $\begin{array}{l}233 \\
29\end{array}$ & $\overline{-}$ & $=$ & 26 & 24 & $\begin{array}{r}10 \\
4\end{array}$ & 1 \\
\hline $22-4,29-33$ & 202.3 & 2 & - & 4 & ${ }_{4}^{2}$ & - & 58 & - & - & 8 & 18 & 4 & 2 \\
\hline $26-4,29-33$ & 240.2 & 5 & - & 21 & 13 & - & 26 & - & - & 31 & 4 & - & - \\
\hline $28-3,12-17$ & 257.1 & - & - & 22 & 3 & - & 19 & - & - & 53 & 3 & $\bar{n}$ & - \\
\hline (3) & $\begin{array}{l}281.6 \\
283.7\end{array}$ & 3 & - & 9 & - & - & 46 & 1 & - & 30 & 1 & 10 & - \\
\hline $\begin{array}{l}31-1,70-75 \\
34, C(16-10)\end{array}$ & $\begin{array}{l}283.7 \\
320.6\end{array}$ & 9 & $\mp$ & 20 & ${ }_{10}^{8}$ & $\bar{z}+$ & 32 & $3^{3}$ & $\bar{z}$ & $\begin{array}{l}20 \\
57\end{array}$ & 10 & 6 & $\bar{z}$ \\
\hline $\begin{array}{l}34, \mathrm{CC}(6-10) \\
40-2,20-22\end{array}$ & $\begin{array}{l}320.6 \\
370.2\end{array}$ & $\begin{array}{l}9 \\
1\end{array}$ & \pm & $\begin{array}{l}15 \\
14\end{array}$ & $\begin{array}{r}10 \\
9\end{array}$ & $\bar{z}$ & $\begin{array}{r}9 \\
22\end{array}$ & $=$ & $\bar{z}$ & $\begin{array}{l}57 \\
6\end{array}$ & $\overline{45}$ & $\overline{3}$ & $=$ \\
\hline Hole 499 & & & & & & & & & & & & & \\
\hline $1-1,142-146$ & 1.4 & 3 & - & 5 & 1 & - & 78 & - & - & 6 & 3 & 4 & \\
\hline $3-2,80-84$ & 12.8 & $\overline{1}$ & 4 & 16 & 2 & + & 28 & 2 & - & 44 & i & 3 & + \\
\hline $\begin{array}{l}5.2,10-14 \\
64,1-5\end{array}$ & $\begin{array}{l}31.1 \\
43.5\end{array}$ & 1 & $\begin{array}{l}6 \\
8\end{array}$ & $\begin{array}{l}18 \\
18 \\
18\end{array}$ & $\begin{array}{l}8 \\
2\end{array}$ & $\begin{array}{r}8 \\
16\end{array}$ & $\begin{array}{l}18 \\
25 \\
25\end{array}$ & $\frac{2}{2}$ & $\bar{z}+x$ & 37 & \pm & - & $\bar{z}+$ \\
\hline 9-1, 83-88 & $\begin{array}{l}48.3 \\
68.3\end{array}$ & $=$ & 8 & $\begin{array}{l}18 \\
17\end{array}$ & - & $\begin{array}{l}16 \\
13\end{array}$ & ${ }_{20}^{23}$ & ${ }_{1}^{2}$ & $\overline{1}$ & ${ }_{34}^{27}$ & s & $\overline{+}$ & 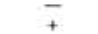 \\
\hline 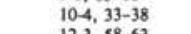 & 81.8 & - & 4 & 30 & - & 10 & 22 & i & - & 32 & - & + & - \\
\hline $12-3,58-63$ & 99.6 & - & 2 & 21 & - & I & 35 & 1 & - & 39 & - & 1 & z \\
\hline $\begin{array}{l}14-2,114-119 \\
15-2,46-48\end{array}$ & $\begin{array}{l}111.6 \\
126.5\end{array}$ & i & $\frac{2}{2}$ & II & $\bar{z}$ & + & $\begin{array}{l}73 \\
45\end{array}$ & 2 & - & $\begin{array}{l}10 \\
30\end{array}$ & $\bar{z}$ & + & $\bar{\dagger}$ \\
\hline $\begin{array}{l}15-2,46-48 \\
18-480-84\end{array}$ & $\begin{array}{l}126.5 \\
158.3\end{array}$ & 1 & ${ }_{2}^{2}$ & ${ }_{6}^{11}$ & $\overline{-}$ & $3^{3}$ & $\begin{array}{l}45 \\
58\end{array}$ & ${ }_{1}^{2}$ & - & $\begin{array}{l}30 \\
1\end{array}$ & $\overline{1}$ & ${ }_{31}^{3}$ & \pm \\
\hline $\begin{array}{l}\begin{array}{l}18-4,4,80-84 \\
23-1,\end{array} 100-104\end{array}$ & $\begin{array}{l}\begin{array}{l}1588.3 \\
2009\end{array}\end{array}$ & $=$ & $\frac{2}{2}$ & ${ }_{2}^{6}$ & $\bar{z}$ & $\overline{1}$ & $\begin{array}{l}38 \\
45\end{array}$ & $i$ & $\mp$ & $\frac{1}{3}$ & - & 46 & $\overline{-}$ \\
\hline $25-4,94-98$ & & + & i & + & - & i & 5 & + & - & 1 & - & 90 & - \\
\hline $28-1,44-49$ & & - & i & 1 & - & + & 46 & - & + & - & - & 51 & - \\
\hline Hole 499B & & & & & & & & & & & & & \\
\hline $4-1,59-64$ & 230.0 & - & - & - & + & - & + & + & - & 5 & $\ddot{\ddot{\theta}}$ & 94 & - \\
\hline $6-1,72-77$ & 249.3 & s & $\overline{7}$ & 1 & - & - & + & - & - & 6 & $\ddot{*}$ & 92 & - \\
\hline $\begin{array}{l}8 \cdot 3,10-14 \\
9, \operatorname{cc}(25-27)\end{array}$ & $\begin{array}{l}270.6 \\
278.8\end{array}$ & $\begin{array}{l}5 \\
6\end{array}$ & ? & ${ }_{2}^{2}$ & $\bar{z}$ & $\begin{array}{l}65 \\
25\end{array}$ & $\begin{array}{l}5 \\
2\end{array}$ & $\Xi$ & \pm & ${ }_{58}^{2} \mathrm{~b}$ & $\ddot{*}$ & $\begin{array}{l}12 \\
2\end{array}$ & $\bar{z}$ \\
\hline Hole 500 & & & & & & & & & & & & & \\
\hline $17-1,123-127$ & 148.2 & 3 & 7 & 3 & + & 69 & 8 & + & + & ? & $\ddot{0}$ & 2 & - \\
\hline $17 . \mathrm{CC}(18-20)$ & 156.2 & - & + & 2 & - & 84 & 3 & - & - & $8^{b}$ & $\cdot \cdot$ & 3 & - \\
\hline
\end{tabular}

$96 \%$ of the $<2-\mu \mathrm{m}$ fraction, except for a sample from Unit 2, where it is $66 \%$ ). Kaolinite + chlorite occurs in Units 1,2 , and $3(3 \%-20 \%$ in the $<2-\mu \mathrm{m}$ fraction), but is virtually absent below, in Upper Cretaceous to lower Miocene sediments. Mica, on the contrary, is absent in upper layers of Unit 1, occurs in trace amounts in its lower layers, and increases in Units 2 to $5(3 \%-14 \%)$. Richest in both mica and kaloinite + chlorite is Sample 494A-17-1, 133-137 cm from Unit 2 (Pliocene). We found only traces of mixed-layer minerals.

The coarse silt fraction mineralogy is also rather uniform. (Fig. 1). In heavy fraction pyroxenes strongly pre- 
Table 2. Mineral composition of sediments, X-ray diffraction data, Site 494.

\begin{tabular}{|c|c|c|c|c|c|c|c|c|c|c|c|c|c|}
\hline \multirow{3}{*}{$\begin{array}{c}\text { Sample } \\
\text { (core-section, } \\
\text { interval in cm) }\end{array}$} & \multirow[b]{3}{*}{$\begin{array}{l}\text { Sub-bottom } \\
\text { Depth (m) }\end{array}$} & \multirow[b]{3}{*}{ Unit } & \multicolumn{11}{|c|}{ Clay Minerals } \\
\hline & & & \multicolumn{3}{|c|}{ Smectite } & \multicolumn{3}{|c|}{ Mica } & \multicolumn{3}{|c|}{$\begin{array}{l}\text { Kaolinite- } \\
\text { Chlorite }\end{array}$} & \multicolumn{2}{|c|}{$z^{b}$} \\
\hline & & & $\begin{array}{l}(<2) \\
(\mu \mathrm{m})\end{array}$ & $\begin{array}{l}(2-20) \\
(\mu \mathrm{m})\end{array}$ & $\begin{array}{c}(>20) \\
(\mu \mathrm{m})\end{array}$ & $\begin{array}{l}(<2) \\
(\mu \mathrm{m})\end{array}$ & $\begin{array}{c}(2-20) \\
(\mu \mathrm{m})\end{array}$ & $\begin{array}{c}(>20) \\
(\mu \mathrm{m})\end{array}$ & $\begin{array}{l}(<2) \\
(\mu \mathrm{m})\end{array}$ & $\begin{array}{c}(2-20) \\
(\mu \mathrm{m})\end{array}$ & $\begin{array}{c}(>20) \\
(\mu \mathrm{m})\end{array}$ & $\begin{array}{l}(<2) \\
(\mu \mathrm{m})\end{array}$ & $\begin{array}{c}(2-20) \\
(\mu \mathrm{m})\end{array}$ \\
\hline \multicolumn{14}{|l|}{ Hole 496} \\
\hline $3-1,50-54$ & 22.26 & 1 & 81 & 81 & 72 & $\operatorname{tr}$ & 10 & 14 & 19 & 9 & 14 & 0.3 & 0.4 \\
\hline $6-4,86-91$ & 50.86 & & 74 & & & 11 & & & 15 & & & 0.4 & \\
\hline $8-2,114-119$ & 67.14 & & 85 & 82 & & 5 & 9 & & 10 & 9 & & 0.5 & 0.4 \\
\hline $19-2,137-139$ & 171.87 & & 80 & 84 & 83 & 6 & 7 & - & 14 & 9 & 17 & 0.5 & 0.5 \\
\hline $22-4,28-33$ & 202.28 & & 76 & & & 9 & & & 15 & & & 0.5 & \\
\hline $26-4,22-27$ & 240.22 & 2 & 81 & 82 & & 8 & 7 & & 11 & 11 & & 0.5 & 0.5 \\
\hline $28-3,12-17$ & 257.62 & & 76 & & & 12 & & & 12 & & & 0.4 & \\
\hline $30-6,58-62$ & 281.58 & & 76 & & & 7 & & & 17 & & & 0.6 & \\
\hline $31-1,70-75$ & 283.70 & & 80 & 85 & & 9 & 5 & & 11 & 10 & & 0.4 & 0.5 \\
\hline $34 C C, 6-10^{a}$ & 320.56 & & 75 & & & 6 & & & 19 & & & 0.6 & \\
\hline $40-2,20-22$ & 370.20 & & 89 & 86 & 80 & 2 & 3 & 2 & 9 & 11 & 18 & 0.5 & 0.7 \\
\hline
\end{tabular}

Note: $++=$ abundant; $+=$ moderate; $\operatorname{tr}=$ trace; $-=$ not found.

a Sample test with $10 \% \mathrm{HCl} 1$ hour at $90^{\circ}-100^{\circ} \mathrm{C}$.

b Coefficient $\mathrm{Z}$ indicates the ratio of $17 \AA$-peak shoulders (see text for an explanation).

dominate over other transparent minerals (75\%-89\%); among these clinopyroxene prevails over orthopyroxene. The third common mineral is hornblende $(8 \%$ $25 \%)$. Epidote content is low $(0 \%-2 \%)$, and other heavy minerals occur sporadically $(1 \%-2 \%$ or traces). In light fraction, the dominant minerals are andesine-labradorite and colorless acidic volcanic glass, whereas quartz content is much lower. In Unit 1 the volcanic glass content is higher relative to feldspar and quartz than in the units below, thus indicating increasing volcaniclastic input during the Pliocene to the Pleistocene. That increase may arise from the activization of andesitic belt volcanism in Central America. Predominance of feldspar over quartz was confirmed by X-ray data (Table 2). The clastic mineral assemblage is typical of the circum-Pacific andesite belt and is one of minor admixture of continental minerals, such as quartz, potassium feldspar, epidote, and acidic rock accessories (Murdmaa et al., 1979).

Authigenic minerals include pyrite, clinoptilolite, glauconite, and gypsum. The pyrite content in coarse silt, heavy fraction (Fig. 1) is lowest in the upper Pleistocene, increases in the lower part of Unit 1, and is almost $100 \%$ in Units 3 through 5, where we found only rare grains of transparent heavy minerals. The downward increase in pyrite is relative and is caused by the decrease in other heavy minerals and total coarse fraction, although we noted some increase in pyrite in smear slides and thin sections as well. Pyrite occurs as microglobules, which can form microscopic chains or crack fillings, and commonly as cubic crystals in the lower part of the section.

As shown by X-ray data, clinoptilolite occurs in all size fractions including finest throughout the section down to 240 meters sub-bottom (Table 2). X-ray and thin section analysis failed to reveal its presence in upper Cretaceous and middle Eocene sediments. We observed rare glauconite grains in most thin sections. Gypsum we observed in a thin section of Sample 494A-17-1, 133$137 \mathrm{~cm}$, where it occurs as fibrous crystals. Plant debris occurs throughout but is unevenly distributed, being most abundant in Quaternary and Pliocene sediments.

\section{Site 496}

Site 496 was drilled on the upper continental slope of the Trench, at a water depth of 2050 meters, $47 \mathrm{~km}$ from the Trench axis and 4000 meters above the Trench floor. A single hole penetrated 378 meters of Quaternary to lower Miocene sediments, represented by diatomaceous mud, sandy mud, and mudstone. We studied 11 samples from 22 meters to 370 meters sub-bottom depth, which represent both lithologic units described in the site chapter (this volume).

The sediments at Site 496 are coarser and less uniform than those at Site 494 (see site chapters, this volume). As expected, terrigenous silt and sand are more abundant, given the relative proximity of the site to land. The sediments contain several ash layers, abundant glauconite, and probably lignite. A thin section from Unit 2, 240 meters sub-bottom, represents diatomaceous sandy mud enriched in micritic carbonate. About $20 \%$ of the total sediment composition consists of clastic minerals, mainly plagioclase. Glauconite is abundant and occurs as micronodules 0.1 to $1.0 \mathrm{~mm}$ in diameter. These are green, light green, or irregularily stained, and their contours are rough and broken by syneresis cracks. The large micronodules contain clay and sand inclusions. We also observed some large irregular forms of a gypsumlike mineral and pyrite microglobules, as well as clinoptilolite crystals 0.07 to 0.02 $\mathrm{mm}$ in size.

X-ray data (Table 3) show a mineral composition of size fractions similar to that at Site 494 except for higher feldspar content. Smectite predominates among clay minerals in all size fractions throughout the section $(72 \%-89 \%)$, but mica and kaolinite contents are somewhat higher than those at Site 494 (Table 3, Fig. 2). Chlorite is certainly detected only in the lower part of 
Table 2. (Continued).

\begin{tabular}{|c|c|c|c|c|c|c|c|c|c|c|c|c|c|c|c|c|}
\hline & & \multicolumn{15}{|c|}{ Other Minerals } \\
\hline \multicolumn{2}{|c|}{$\mathrm{z}^{\mathrm{b}}$} & \multicolumn{3}{|c|}{ Quartz } & \multicolumn{3}{|c|}{ Feldspar } & \multicolumn{3}{|c|}{ Clinoptilolite } & \multicolumn{3}{|c|}{ Calcite } & \multicolumn{3}{|c|}{ Amorphous Phase } \\
\hline $\begin{array}{c}(2-20) \\
(\mu \mathrm{m})\end{array}$ & $\begin{array}{c}(>20) \\
(\mu \mathrm{m})\end{array}$ & $\begin{array}{l}(<2) \\
(\mu \mathrm{m})\end{array}$ & $\begin{array}{c}(2-20) \\
(\mu \mathrm{m})\end{array}$ & $\begin{array}{c}(>20) \\
(\mu \mathrm{m})\end{array}$ & $\begin{array}{l}(<2) \\
(\mu \mathrm{m})\end{array}$ & $\begin{array}{c}(2-20) \\
(\mu \mathrm{m})\end{array}$ & $\begin{array}{c}(>20) \\
(\mu \mathrm{m})\end{array}$ & $\begin{array}{l}(<2) \\
(\mu \mathrm{m})\end{array}$ & $\begin{array}{c}(2-20) \\
(\mu \mathrm{m})\end{array}$ & $\begin{array}{c}(>20) \\
(\mu \mathrm{m})\end{array}$ & $\begin{array}{l}(<2) \\
(\mu \mathrm{m})\end{array}$ & $\begin{array}{c}(2-20) \\
(\mu \mathrm{m})\end{array}$ & $\begin{array}{c}(>20) \\
(\mu \mathrm{m})\end{array}$ & $\begin{array}{l}(<2) \\
(\mu \mathrm{m})\end{array}$ & $\begin{array}{c}(2-20) \\
(\mu \mathrm{m})\end{array}$ & $\begin{array}{c}(>20) \\
(\mu \mathrm{m})\end{array}$ \\
\hline 0.5 & 0.4 & $\begin{array}{l}\mathrm{tr} \\
\mathrm{tr} \\
\mathrm{tr}\end{array}$ & tr & + & $\begin{array}{l}+ \\
+ \\
\text { tr }\end{array}$ & + & + & $\begin{array}{l}\text { tr } \\
\text { tr } \\
\text { tr }\end{array}$ & tr & $\operatorname{tr}$ & $\bar{z}$ & - & - & $\begin{array}{l}\text { tr } \\
\text { tr } \\
\text { tr }\end{array}$ & tr & tr \\
\hline 0.4 & & tr & tr & & + & ++ & & - & $\operatorname{tr}$ & & - & - & & tr & tr & \\
\hline 0.5 & & $\mathrm{tr}$ & tr & & + & ++ & & $\mathrm{tr}$ & tr & & - & - & & tr & tr & \\
\hline 0.6 & & tr & tr & & + & ++ & & $\mathrm{tr}$ & $\mathrm{tr}$ & & - & - & & tr & tr & \\
\hline- & & tr & tr & & $\mathrm{tr}$ & ++ & & - & - & & - & - & & tr & tr & \\
\hline 0.5 & 0.4 & $\mathrm{tr}$ & & & $\mathrm{tr}$ & & & - & & & - & & & tr & & \\
\hline- & - & $\begin{array}{l}\mathrm{tr} \\
\mathrm{tr}\end{array}$ & tr & + & $\begin{array}{r}\text { tr } \\
+\end{array}$ & + & + & tr & + & + & $\bar{z}$ & - & - & $\begin{array}{l}\mathrm{tr} \\
\mathrm{tr}\end{array}$ & tr & tr \\
\hline- & - & $\begin{array}{l}\mathrm{tr} \\
\mathrm{tr}\end{array}$ & - & - & $\begin{array}{l}\text { tr } \\
+\end{array}$ & - & - & $\overline{-}$ & - & - & + & ++ & ++ & $\begin{array}{l}\mathrm{tr} \\
\mathrm{tr}\end{array}$ & - & - \\
\hline
\end{tabular}

A

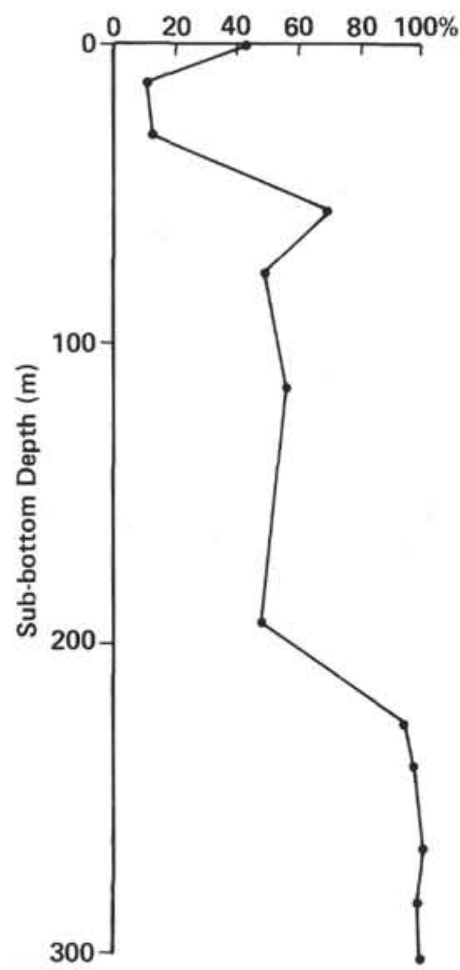

B

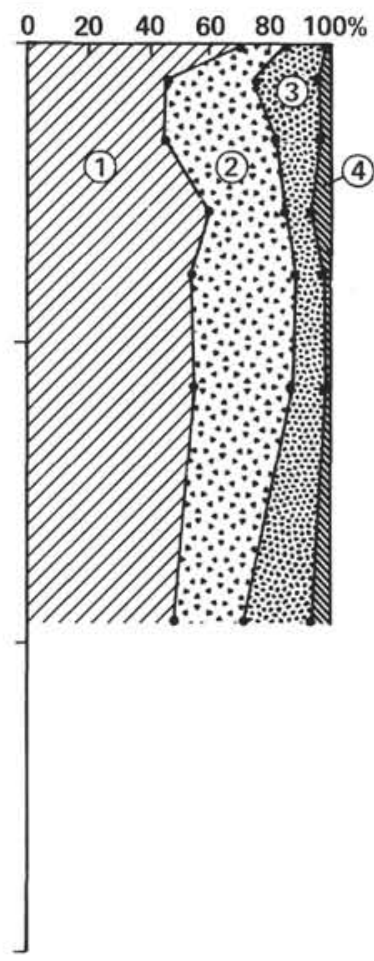

C

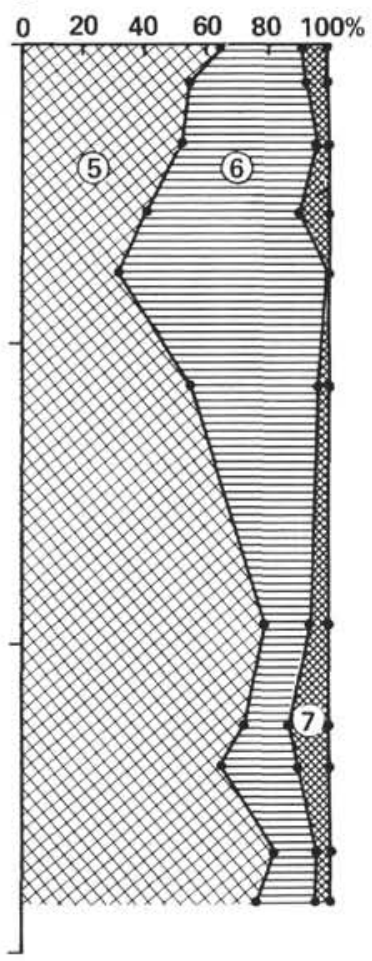

D

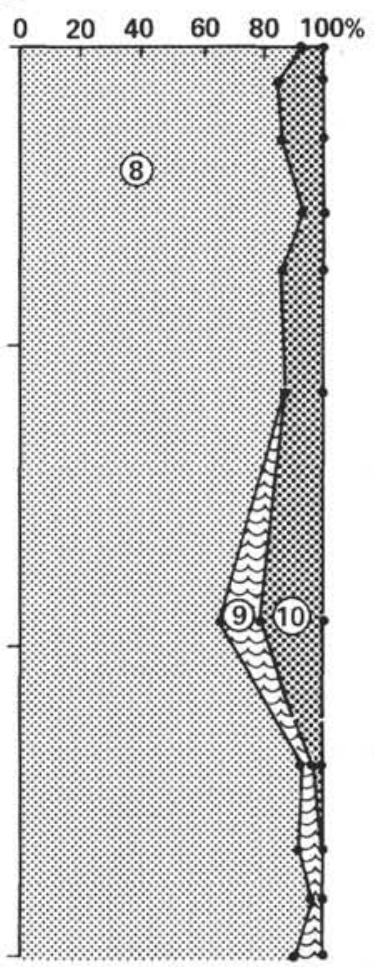

Figure 1. Changes in sediment mineralogy with sub-bottom depth at Site 494. (A. Pyrite content in heavy fractions of coarse silt. B. Transparent minerals in heavy fraction: $1=$ clinopyroxene; $2=$ orthopyroxene; $3=$ hornblende; and $4=$ others. C. Light fraction: $5=$ volcanic glass; $6=$ feldspar; $7=$ quartz. D. Clay minerals in $<2-\mu \mathrm{m}$ fraction: $8=$ smectite; $9=$ mica; $10=$ kaolinite + chlorite.)

the section (Cores 34 and 40). In Sample 496-31-1, $70-75 \mathrm{~cm}$, a minor admixture of halloysite is likely present, as it is at Site 494 (Sample 494A-20-5, 46-56 cm). On the electron photomicrographs of these two samples we observed short tubular particles; these particles may be halloysite.

The heavy coarse silt fraction is uniform throughout the section studied down to 284 meters sub-bottom depth. The two lowermost samples (Cores 34 and 40) are composed totally of authigenic minerals, aggregates of a ferruginous carbonate and pyrite. Three transparent heavy minerals-clinopyroxene, orthopyroxene, and hornblende-predominate. Only rare grains of garnet were found in a sample from Core 6, and epidote is present in a sample from Core 31 . The latter also differs from other samples because of its higher hornblende 
Table 3. Mineral composition of sediments, X-ray diffraction data, Site 496.

\begin{tabular}{|c|c|c|c|c|c|c|c|c|c|c|c|c|c|}
\hline \multirow{3}{*}{$\begin{array}{c}\text { Sample } \\
\text { (core-section, } \\
\text { interval in } \mathrm{cm} \text { ) }\end{array}$} & \multirow[b]{3}{*}{$\begin{array}{c}\text { Sub-bottom } \\
\text { Depth (m) }\end{array}$} & \multirow[b]{3}{*}{ Unit } & \multicolumn{11}{|c|}{ Clay Minerals } \\
\hline & & & \multicolumn{3}{|c|}{ Smectite } & \multicolumn{3}{|c|}{ Mica } & \multicolumn{3}{|c|}{$\begin{array}{c}\text { Kaolinite- } \\
\text { Chlorite }\end{array}$} & \multicolumn{2}{|c|}{$z^{b}$} \\
\hline & & & $\begin{array}{l}(<2) \\
(\mu \mathrm{m})\end{array}$ & $\begin{array}{c}(2-20) \\
(\mu \mathrm{m})\end{array}$ & $\begin{array}{c}(>20) \\
(\mu \mathrm{m})\end{array}$ & $\begin{array}{l}(<2) \\
(\mu \mathrm{m})\end{array}$ & $\begin{array}{c}(2-20) \\
(\mu \mathrm{m})\end{array}$ & $\begin{array}{c}(>20) \\
(\mu \mathrm{m})\end{array}$ & $\begin{array}{l}(<2) \\
(\mu \mathrm{m})\end{array}$ & $\begin{array}{c}(2-20) \\
(\mu \mathrm{m})\end{array}$ & $\begin{array}{c}(>20) \\
(\mu \mathrm{m})\end{array}$ & $\begin{array}{l}(<2) \\
(\mu \mathrm{m})\end{array}$ & $\begin{array}{c}(2-20) \\
(\mu \mathrm{m})\end{array}$ \\
\hline \multicolumn{14}{|l|}{ Hole 496} \\
\hline $\begin{array}{l}3-1,50-54 \\
6-4,86-91\end{array}$ & $\begin{array}{l}22.26 \\
50.86\end{array}$ & 1 & $\begin{array}{l}81 \\
74\end{array}$ & 81 & 72 & $\begin{array}{l}\mathrm{tr} \\
11\end{array}$ & 10 & 14 & $\begin{array}{l}19 \\
15\end{array}$ & 9 & 14 & $\begin{array}{l}0.3 \\
0.4\end{array}$ & 0.4 \\
\hline $8-2,114-119$ & 67.14 & & 85 & 82 & & 5 & 9 & & 10 & 9 & & 0.5 & 0.4 \\
\hline $19-2,137-139$ & 171.87 & & 80 & 84 & 83 & 6 & 7 & - & 14 & 9 & 17 & 0.5 & 0.5 \\
\hline $22-4,28-33$ & 202.28 & & 76 & & & 9 & & & 15 & & & 0.5 & \\
\hline $26-4,22-27$ & 240.22 & 2 & 81 & 82 & & 8 & 7 & & 11 & 11 & & 0.5 & 0.5 \\
\hline $28-3,12-17$ & 257.62 & & 76 & & & 12 & & & 12 & & & 0.4 & \\
\hline $30-6,58-62$ & 281.58 & & 76 & & & 7 & & & 17 & & & 0.6 & \\
\hline $31-1,70-75$ & 283.70 & & 80 & 85 & & 9 & 5 & & 11 & 10 & & 0.4 & 0.5 \\
\hline $34 C C, 6-10^{a}$ & 320.56 & & 75 & & & 6 & & & 19 & & & 0.6 & \\
\hline $40-2,20-22$ & 370.20 & & 89 & 86 & 80 & 2 & 3 & 2 & 9 & 11 & 18 & 0.5 & 0.7 \\
\hline
\end{tabular}

Note: $++=$ abundant; $+=$ moderate; $\operatorname{tr}=$ trace; $-=$ not found.

a Sample test with $10 \% \mathrm{HCl} 1$ hour at $90^{\circ}-100^{\circ} \mathrm{C}$.

b Coefficient $Z$ indicates the ratio of $17 \AA \AA$-peak shoulders (see text for an explanation).

content, thus showing a more "continental" composition.

The light fraction is more variable than that at Site 494 and contains more quartz, especially in the lower part of the section (Core 34). Labradorite and colorless volcanic glass predominate throughout, but their interrelations are variable: in Unit 1 plagioclase predominates, whereas in Unit 2 its proportion is close to that of glass. Along with glass, abundant lithic ash occurs in the light fraction. The mineral assemblage, on the whole, corresponds to the andesitic-belt type, mentioned earlier in the discussion of Site 494 .

\section{Site 495}

This site is located on the eastern margin of the Cocos Plate $12 \mathrm{~km}$ seaward of the Trench axis, at a water depth of 4140 meters and on an elevated ridge. The hole was drilled to a sub-bottom depth of 446.5 meters, penetrating 428.5 meters of Miocene to Quaternary sediments above basaltic basement. The upper 171 meters are composed of upper Pliocene to Quaternary hemipelagic diatomaceous mud, overlying about 7 meters of middle Miocene brown pelagic clay. Below a sub-bottom depth of 178 meters, drilling recovered lower to middle Miocene chalk with chert, the lowermost 22 meters being enriched in iron and manganese. We studied samples from each of these lithologic types.

Several thin sections from Unit 1 contain diatomaceous mud composed of diatom frustules and their fragments, with a minor admixture of radiolarians, sponge spicules, and nannofossils mounted in a smectitic clay matrix. Silt and sand-size clastic minerals, quartz, plagioclase, and rare biotite occur as minor constituents, much less abundant than on the continental slope. Pyrite occurs throughout; we found clinoptilolite only in Core 3 . In a thin section from Core 18 , sediment is irregularly stained by iron oxides, like the pelagic clay of Unit 2 .

$\mathrm{X}$-ray data show that smectite strongly predominates over other clay minerals $(83 \%-100 \%$, Table 4 , Fig. 3$)$.
Mica, chlorite, and kaolinite were also detected in most samples. Mica content is low, occurring in traces and up to $9 \%$ except for the two lowest Unit 1 samples, where it is $12 \%$ and $17 \%$ (Table 4 ). Because of its seaward location feldspar, quartz, and kaolinite contents are markedly lower at Site 495 than at Sites 494 and 496, as expected. We detected traces of clinoptilolite in most samples from Unit 2, and in several cases it is associated with cristobalite.

The total coarse silt fraction, as well as the heavy fraction, are less abundant than in hemipelagic sediments from the continental slope. The low heavy mineral content most likely results from irregular variations of transparent minerals counted. Anyway, clinopyroxene predominates in most samples, hornblende content is commonly higher, and orthopyroxene content is lower than in hemipelagic sediments from Sites 494 and 496. Pyrite content is variable, showing an irregular but decreasing trend downhole below 100 meters sub-bottom depth (Fig. 3).

The light fraction is characterized by high volcanic glass content and apparent domination of labradoritic plagioclase over quartz. The sediments differ from those of the continental slope because of their extremely low quartz content.

A single sample of pelagic clay from Unit 2 (Core 19), examined in thin section, is a radiolarian clay irregularly stained by iron oxides. Clastic minerals are rare and clinoptilolite was not found. Smectite predominates among clay minerals $(85 \%)$, and the kaolinite + chlorite content is $10 \%$, somewhat more than in Unit 1 . Only 28 grains of transparent heavy minerals were found in coarse fraction, and those were represented by pyroxenes. The light fraction is composed almost totally of colorless volcanic glass.

Calcareous sediments of Unit 3 are composed of nannofossils with a considerable amount of radiolarians, partly replaced by calcite. Terrigenous clastic minerals are virtually absent. X-ray data show traces of smectite 
Table 3. (Continued).

\begin{tabular}{|c|c|c|c|c|c|c|c|c|c|c|c|c|c|c|c|}
\hline \multirow{3}{*}{$\begin{array}{l}\text { Clay } \\
\text { Minerals } \\
\frac{\mathrm{Z}^{\mathrm{b}}}{(>20)} \\
(\mu \mathrm{m})\end{array}$} & \multicolumn{15}{|c|}{ Other Minerals } \\
\hline & \multicolumn{3}{|c|}{ Quartz } & \multicolumn{3}{|c|}{ Feldspar } & \multicolumn{3}{|c|}{ Clinoptilolite } & \multicolumn{3}{|c|}{ Calcite } & \multicolumn{3}{|c|}{ Amorphous Phase } \\
\hline & $\begin{array}{l}(<2) \\
(\mu \mathrm{m})\end{array}$ & $\begin{array}{c}(2-20) \\
(\mu \mathrm{m})\end{array}$ & $\begin{array}{c}(>20) \\
(\mu \mathrm{m})\end{array}$ & $\begin{array}{l}(<2) \\
(\mu \mathrm{m})\end{array}$ & $\begin{array}{c}(2-20) \\
(\mu \mathrm{m})\end{array}$ & $\begin{array}{c}(>20) \\
(\mu \mathrm{m})\end{array}$ & $\begin{array}{l}(<2) \\
(\mu \mathrm{m})\end{array}$ & $\begin{array}{c}(2-20) \\
(\mu \mathrm{m})\end{array}$ & $\begin{array}{c}(>20) \\
(\mu \mathrm{m})\end{array}$ & $\begin{array}{l}(<2) \\
(\mu \mathrm{m})\end{array}$ & $\begin{array}{c}(2-20) \\
(\mu \mathrm{m})\end{array}$ & $\begin{array}{c}(>20) \\
(\mu \mathrm{m})\end{array}$ & $\begin{array}{l}(<2) \\
(\mu \mathrm{m})\end{array}$ & $\begin{array}{c}(2-20) \\
(\mu \mathrm{m})\end{array}$ & $\begin{array}{c}(>20) \\
(\mu \mathrm{m})\end{array}$ \\
\hline \multirow[t]{2}{*}{0.4} & $\begin{array}{l}\mathrm{tr} \\
\mathrm{tr}\end{array}$ & $\mathrm{tr}$ & $\operatorname{tr}$ & $\begin{array}{l}+ \\
+\end{array}$ & ++ & + & $\overline{\operatorname{tr}}$ & $\mathrm{tr}$ & - & - & - & - & $\begin{array}{l}\mathrm{tr} \\
\mathrm{tr}\end{array}$ & $\operatorname{tr}$ & $\mathrm{tr}$ \\
\hline & tr & $\operatorname{tr}$ & & + & + & & $\mathrm{tr}$ & $\operatorname{tr}$ & & - & - & & $\mathrm{tr}$ & tr & \\
\hline \multirow[t]{6}{*}{0.6} & $\begin{array}{l}\text { tr } \\
\text { tr }\end{array}$ & tr & $\operatorname{tr}$ & $\underset{\mathrm{tr}}{++}$ & ++ & ++ & $\begin{array}{ll}\mathrm{tr} \\
\mathrm{tr}\end{array}$ & $\operatorname{tr}$ & - & - & - & + & $\begin{array}{l}\mathrm{tr} \\
\mathrm{tr}\end{array}$ & tr & $\operatorname{tr}$ \\
\hline & $\mathrm{tr}$ & $\operatorname{tr}$ & & + & ++ & & $\operatorname{tr}$ & $\mathrm{tr}$ & & - & - & & $\operatorname{tr}$ & $\operatorname{tr}$ & \\
\hline & tr & & & + & & & - & & & - & & & tr & & \\
\hline & tr & & & + & & & $\operatorname{tr}$ & & & - & & & $\mathrm{tr}$ & & \\
\hline & tr & tr & & tr & + & & $\operatorname{tr}$ & tr & & - & - & & tr & tr & \\
\hline & tr & & & + & & & - & & & - & & & $\mathrm{tr}$ & & \\
\hline 0.7 & tr & tr & tr & + & + & + & - & $\mathrm{tr}$ & - & - & - & + & $\mathrm{tr}$ & tr & tr \\
\hline
\end{tabular}
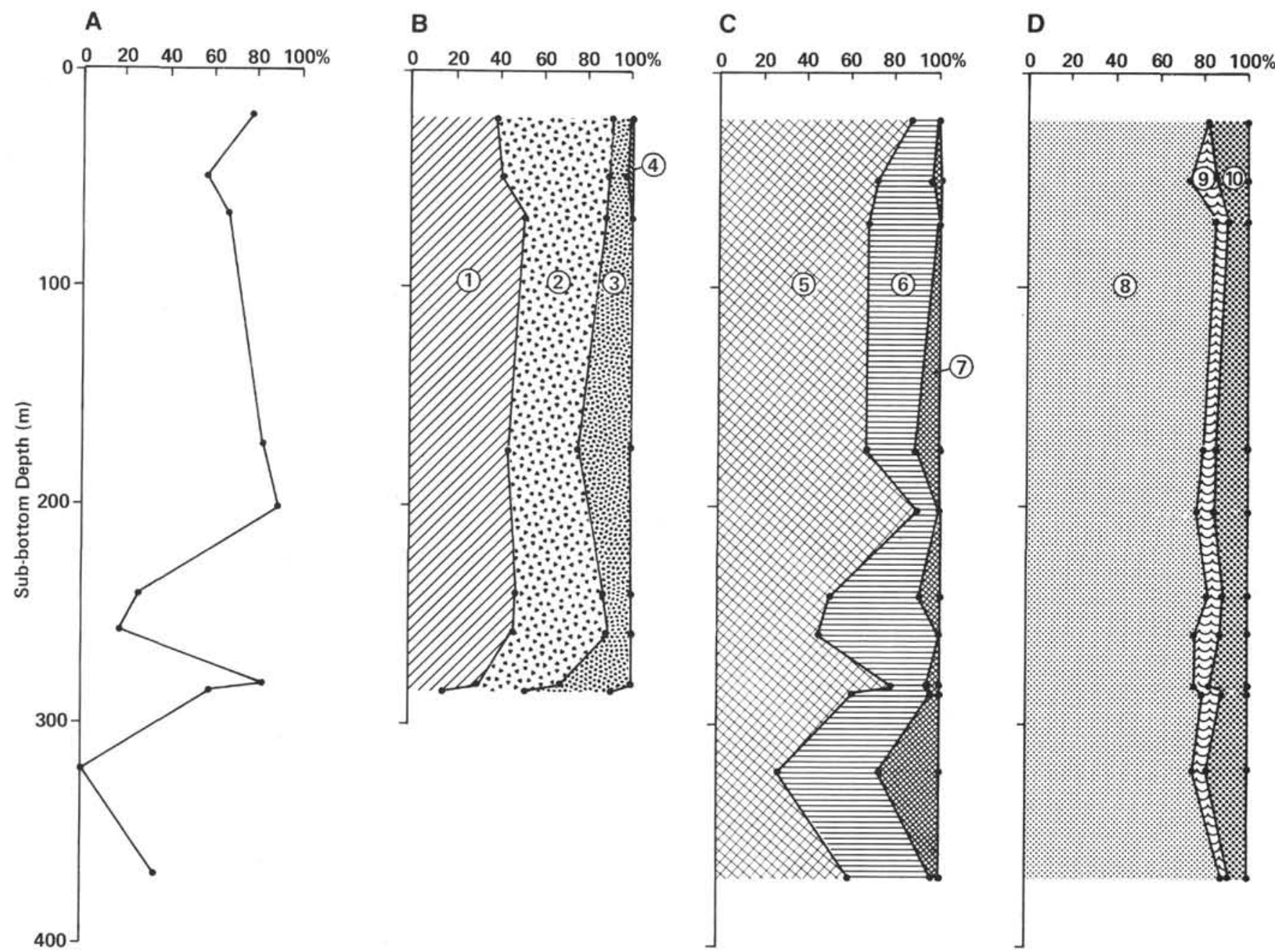

Figure 2. Changes in mineralogy of sediments with sub-bottom depth for Site 496. (Graphic symbols are explained in the caption for Fig. 1.) 
Table 4. Mineral composition of sediments, X-ray diffraction data, Site 495 .

\begin{tabular}{|c|c|c|c|c|c|c|c|c|c|c|c|c|c|}
\hline \multirow{3}{*}{$\begin{array}{c}\text { Sample } \\
\text { (core-section, } \\
\text { interval in } \mathrm{cm} \text { ) }\end{array}$} & \multirow[b]{3}{*}{$\begin{array}{l}\text { Sub-bottom } \\
\text { Depth (m) }\end{array}$} & \multirow[b]{3}{*}{ Unit } & \multicolumn{11}{|c|}{ Clay Minerals } \\
\hline & & & \multicolumn{3}{|c|}{ Smectite } & \multicolumn{3}{|c|}{ Mica } & \multicolumn{3}{|c|}{$\begin{array}{c}\text { Kaolinite + } \\
\text { Chlorite }\end{array}$} & \multicolumn{2}{|c|}{$z^{b}$} \\
\hline & & & $\begin{array}{l}(<2) \\
(\mu \mathrm{m})\end{array}$ & $\begin{array}{c}(2-20) \\
(\mu \mathrm{m})\end{array}$ & $\begin{array}{c}(>20) \\
(\mu \mathrm{m})\end{array}$ & $\begin{array}{l}(<2) \\
(\mu \mathrm{m})\end{array}$ & $\begin{array}{c}(2-20) \\
(\mu \mathrm{m})\end{array}$ & $\underset{(\mu \mathrm{m})}{(>20)}$ & $\begin{array}{l}(<2) \\
(\mu \mathrm{m})\end{array}$ & $\begin{array}{c}(2-20) \\
(\mu \mathrm{m})\end{array}$ & $\underset{(\mu \mathrm{m})}{(>20)}$ & $\begin{array}{l}(<2) \\
(\mu \mathrm{m})\end{array}$ & $\begin{array}{l}(2-20) \\
(\mu \mathrm{m})\end{array}$ \\
\hline \multicolumn{14}{|l|}{ Hole 496} \\
\hline $3-1,42-47$ & 19.42 & 1 & 86 & 100 & $\operatorname{tr}$ & 5 & - & - & 9 & - & - & 0.4 & 0.6 \\
\hline $4-1,44-48$ & 28.94 & & 85 & & & 6 & & & 9 & & & 0.6 & \\
\hline $4-5,110-114$ & 35.60 & & 93 & & & 3 & & & 4 & & & 0.5 & \\
\hline $6-4,16-20$ & 52.16 & & 100 & 90 & & $\operatorname{tr}$ & 5 & & $\operatorname{tr}$ & 5 & & 0.6 & 0.6 \\
\hline $7-1,26-30$ & 57.26 & & 92 & & & 5 & & & 3 & & & 0.5 & \\
\hline $9-2,20-25$ & 77.20 & & 91 & 88 & 93 & 6 & 7 & 2 & 3 & 5 & 5 & 0.5 & 0.6 \\
\hline $12-5,21-26$ & 110.71 & & 88 & & & 9 & & & 3 & & & 0.6 & \\
\hline $13-3,56-58$ & 117.56 & & 88 & 96 & & 3 & tr & & 9 & 4 & & 0.5 & 0.6 \\
\hline $15-2,70-72$ & 135.20 & & 85 & & & 12 & & & 3 & & & 0.4 & \\
\hline $18-5,70-72$ & 168.20 & & 83 & 85 & & 17 & 15 & & - & - & & 0.5 & 0.5 \\
\hline $19-4,120-124^{\mathrm{a}}$ & 176.70 & 2 & 85 & & & 5 & & & 10 & & & 0.7 & \\
\hline $20-5,138-142$ & 187.88 & 3 & tr & & & - & & & - & & & & \\
\hline $21-4,40-45$ & 194.90 & & $\mathrm{tr}$ & $\mathrm{tr}$ & $\operatorname{tr}$ & - & - & - & - & - & - & & \\
\hline $22-5,52-57$ & 206.02 & & $\mathrm{tr}$ & & & - & & & - & & & & \\
\hline $24-4,40-42$ & 223.40 & & tr & & & - & & & - & & & & \\
\hline $25-6,10-15$ & 235.60 & & & $\operatorname{tr}$ & & & - & & & - & & & \\
\hline $27-3,7-12$ & 250.07 & & & $\mathrm{tr}$ & & & - & & & - & & & \\
\hline $28-2,30-35$ & 258.30 & & tr & $\mathrm{tr}$ & & - & - & & - & - & & & \\
\hline $28-7,5-9$ & 265.55 & & $\mathrm{tr}$ & & & - & & & - & & & & \\
\hline $29-4,80-82$ & 271.30 & & tr & & & - & & & - & & & & \\
\hline $30-2,20-24^{a}$ & 277.20 & & $\mathrm{tr}$ & & & - & & & - & & & & \\
\hline $31-5,17-21$ & 291.17 & & $\mathrm{tr}$ & & & - & & & - & & & & \\
\hline $32-1,35-39$ & 294.85 & & $\mathrm{tr}$ & & & - & & & - & & & & \\
\hline $33-5,20-25$ & 310.20 & & $\mathrm{tr}$ & & & - & & & - & & & & \\
\hline $35-3,16-20$ & 326.16 & & $\mathrm{tr}$ & $\mathrm{tr}$ & & - & - & & - & - & & & \\
\hline $37-3,51-53$ & 345.51 & & & $\mathrm{tr}$ & & & - & & & - & & & \\
\hline $39-3,50-52^{\mathrm{a}}$ & 364.50 & & $\operatorname{tr}$ & tr & & - & - & & - & - & & & \\
\hline $43-3,49-51$ & 402.49 & & & $\mathrm{tr}$ & & & - & & & - & & & \\
\hline $45-3,52-54$ & 421.50 & & & tr & & & - & & & - & & & \\
\hline $46-1,108-110^{a}$ & 428.58 & & & $\mathrm{tr}$ & & & - & & & - & & & \\
\hline
\end{tabular}

Note: $++=$ abundant; $+=$ moderate; $\mathrm{tr}=$ trace; $-=$ not found.

a Sample test with $10 \% \mathrm{HCl} 1$ hour at $90^{\circ}-100^{\circ} \mathrm{C}$.

$\mathrm{b}$ Coefficient $\mathrm{Z}$ indicates the ratio of $17 \AA$-peak shoulders (see the text for an explanation).

and the absence of other clay minerals and of quartz, feldspar, and clinoptilolite as well (Table 4). The mineralogy of the coarse fraction is variable, but both heavy and light fractions contain relatively more minerals of a "continental" origin than do the overlying units, i.e., hornblende, epidote, garnet, and apatite in heavy fraction, quartz and feldspar in light fraction. The intervals of this terrigenous mineralogy alternate with more volcanogenic ones in the upper portion of the unit and seem to be common in the lower portion. On the whole, however, the total clastic mineral content is very low. Pyrite content is highly variable, as well as its oxidation state.

\section{Sites $\mathbf{4 9 9}$ and $\mathbf{5 0 0}$}

Holes at these sites were drilled on the Trench floor at a water depth of about 6125 meters. We studied samples from Site 499, Unit 1 (Quaternary diatomaceous mud and turbiditic sand), Unit 2 (lower Quaternary and upper Pliocene hemipelagic mud), Unit 4 (lower Miocene nannofossil chalk), and Unit 5 (lower Miocene manganiferous chalk). Three samples from Site 500 represent Units 4 and 5.
A thin section from Core 6 indicates a probable turbidite layer. The sediment is composed of diatoms with a minor amount of radiolarians, foraminifers, and nannofossils, and of terrigenous clay and clastic minerals. Clastic minerals comprise $25 \%$ to $30 \%$ of the total sediment and include feldspar, quartz, biotite, with minor admixture of hornblende, pyroxenes, chlorite, and some accessories. In the thin section we observed a small (2 $\mathrm{mm}$ ) carbonate concretion filled with clay and plant debris, a gypsum aggregate, and abundant microglobular pyrite.

Another thin section from Unit 1 (Core 12, Section 3) represents diatom ooze with rather low silt-size clasticmineral content $(5 \%-8 \%)$ and rare plant debris. We observed crystals of clinoptilolite $(10-20 \mu \mathrm{m})$, microglobular pyrite, and small lenses of gypsum (0.1-0.5 $\mathrm{mm}$ ).

X-ray data show that smectite dominates among the clay minerals $(76 \%-89 \%)$, but with considerable mica (up to 20\%) and kaolinite (up to 15\%) content (Table 5, Fig. 4). We did not recover any chlorite. Feldspars (mainly plagioclase) are common, traces of quartz were detected in all samples, whereas traces of clinoptilolite 
Table 4. (Continued).

\begin{tabular}{|c|c|c|c|c|c|c|c|c|c|c|c|c|c|c|c|}
\hline \multirow{3}{*}{$\begin{array}{c}\begin{array}{c}\text { Clay } \\
\text { Minerals }\end{array} \\
\frac{\mathrm{Z}^{\mathrm{b}}}{(>20)} \\
\begin{array}{c}(\mu \mathrm{m}) \\
(1)\end{array}\end{array}$} & \multicolumn{15}{|c|}{ Other Minerals } \\
\hline & \multicolumn{3}{|c|}{ Quartz } & \multicolumn{3}{|c|}{ Feldspar } & \multicolumn{3}{|c|}{ Clinoptilolite } & \multicolumn{3}{|c|}{ Calcite } & \multicolumn{3}{|c|}{ Amorphous Phase } \\
\hline & $\begin{array}{l}(<2) \\
(\mu \mathrm{m})\end{array}$ & $\begin{array}{c}(2-20) \\
(\mu \mathrm{m})\end{array}$ & $\begin{array}{c}(>20) \\
(\mu \mathrm{m})\end{array}$ & $\begin{array}{l}(<2) \\
(\mu \mathrm{m})\end{array}$ & $\begin{array}{c}(2-20) \\
(\mu \mathrm{m})\end{array}$ & $\begin{array}{c}(>20) \\
(\mu \mathrm{m})\end{array}$ & $\begin{array}{l}(<2) \\
(\mu \mathrm{m})\end{array}$ & $\begin{array}{c}(2-20) \\
(\mu \mathrm{m})\end{array}$ & $\begin{array}{c}(>20) \\
(\mu \mathrm{m})\end{array}$ & $\begin{array}{l}(<2) \\
(\mu \mathrm{m})\end{array}$ & $\begin{array}{c}(2-20) \\
(\mu \mathrm{m})\end{array}$ & $\begin{array}{c}(>20) \\
(\mu \mathrm{m})\end{array}$ & $\begin{array}{l}(<2) \\
(\mu \mathrm{m})\end{array}$ & $\begin{array}{c}(2-20) \\
(\mu \mathrm{m})\end{array}$ & $\underset{(\mu \mathrm{m})}{(>20)}$ \\
\hline & tr & $\operatorname{tr}$ & tr & $\operatorname{tr}$ & + & ++ & $\operatorname{tr}$ & $\operatorname{tr}$ & - & - & - & - & $\operatorname{tr}$ & $\operatorname{tr}$ & + \\
\hline & $\operatorname{tr}$ & & & $\operatorname{tr}$ & & & tr & & & - & & & tr & & \\
\hline & tr & & & tr & & & tr & & & - & & & tr & & \\
\hline & tr & $\operatorname{tr}$ & & tr & + & & - & tr & & - & - & & tr & tr & \\
\hline & tr & & & $\operatorname{tr}$ & & & - & & & - & & & + & & \\
\hline \multirow[t]{17}{*}{0.6} & tr & tr & $\operatorname{tr}$ & tr & + & + & tr & $\operatorname{tr}$ & tr & - & - & - & tr & tr & tr \\
\hline & tr & & & + & t & & tr & & & & & & $\operatorname{tr}$ & tr & \\
\hline & tr & tr & & $\begin{array}{l}\text { tr } \\
\text { tr }\end{array}$ & + & & & tr & & $\bar{z}$ & - & & $\begin{array}{l}\mathrm{tr} \\
\mathrm{tr}\end{array}$ & tr & \\
\hline & $\begin{array}{l}\operatorname{tr} \\
\text { tr }\end{array}$ & tr & & tr & + & & $\frac{\operatorname{tr}}{-}$ & - & & $\bar{z}$ & - & & $\begin{array}{l}\text { tr } \\
+\end{array}$ & + & \\
\hline & tr & & & $\operatorname{tr}$ & & & - & & & - & & & + & & \\
\hline & - & - & - & - & - & & - & & & ++ & + & + & - & - & $\pi$ \\
\hline & - & - & - & $\overline{-}$ & - & - & $\overline{-}$ & - & - & $\begin{array}{l}++ \\
++\end{array}$ & ++ & ++ & $\overline{-}$ & - & - \\
\hline & - & & & - & & & - & & & ++ & & & - & & \\
\hline & & $\overline{-}$ & & & - & & & - & & & $\begin{array}{l}++ \\
++\end{array}$ & & & - & \\
\hline & - & - & & - & - & & - & - & & ++ & ++ & & - & - & \\
\hline & - & & & - & & & - & & & ++ & & & - & & \\
\hline & - & & & - & & & - & & & ++ & & & - & & \\
\hline & - & & & - & & & - & & & ++ & & & - & & \\
\hline & - & & & - & & & - & & & ++ & & & - & & \\
\hline & - & & & - & & & - & & & ++ & & & - & & \\
\hline & - & & & - & - & & - & - & & $\begin{array}{l}++ \\
++\end{array}$ & ++ & & $\overline{-}$ & - & \\
\hline & & - & & & - & & - & $\bar{z}$ & & ++ & $\begin{array}{l}+1 \\
++\end{array}$ & & - & $\overline{-}$ & \\
\hline & - & - & & - & - & & - & - & & ++ & ++ & & - & - & \\
\hline & & $\overline{-}$ & & & - & & & - & & & ++ & & & - & \\
\hline & & $\overline{-}$ & & & $\overline{-}$ & & & $\overline{-}$ & & & $\begin{array}{l}++ \\
++\end{array}$ & & & $\overline{-}$ & \\
\hline
\end{tabular}

and cristobalite occur in half of them, without any regular distribution pattern.

The coarse-silt heavy fraction from Unit 1 is similar to that at Site 494, but contains somewhat more hornblende and epidote. The light fraction composition is almost identical to that at Site 494 and differs markedly from that at Site 495 . The light fractions at Sites 494 and 499 are characterized by higher feldspar and moderate volcanic glass content, whereas volcanic glass strongly predominates in hemipelagic noncalcareous sediments from Site 495.

Unit 2, described as hemipelagic mud, is quite similar to Unit 1 according to our mineralogical and thin-section data. On the basis of a thin section from Core 23, this unit contains abundant siliceous microfossils in a clay matrix that includes a few fine grains of feldspar and quartz. X-ray data show somewhat higher smectite content (85\%-92\% in clay fraction) and less mica, kaolinite, and feldspar than in Unit 1 . We found clinoptilolite in each of four samples studied. The transition from Unit 1 to Unit 2 is marked in coarse silt composition by the decrease in hornblende, epidote, and biotite (heavy fraction), and by the decrease in feldspar relative to volcanic glass (light fraction).
Lower Miocene calcareous sediments, nannofossil chalk and nannofossil manganiferous chalk, are poorly characterized by our mineralogical study, due to high calcite content, which masks all other minerals. Thin sections and smear slides show, and coarse fraction mineralogy confirms, however, that both lithologic varieties of the chalk contain considerable amounts of siliceous microfossils (Table 1). These are mainly radiolarians, but some diatoms and spicules do occur.

The manganiferous chalk contains abundant ferromanganese micronodules, which we observed both in thin sections and in the light fraction of the coarse silt (Table 1). Very low heavy fraction content prevented us from obtaining reliable quantitative data, but in several heavy fractions authigenic barite apparently predominates over other transparent minerals.

$\mathrm{X}$-ray data show in all samples only abundant calcite and traces of smectite, and in one case traces of mica. Smectite from a sample of manganiferous chalk from Hole 499B, Core 9,CC is ribbon-shaped, whereas that from Hole 500, Core 17, Section 1 is fibrous (see Plate 1), thus showing the characteristic morphology of authigenic Fe-montmorillonite (Kossovskaya, 1975), which was also confirmed by test with hot $10 \% \mathrm{HCl}$. 

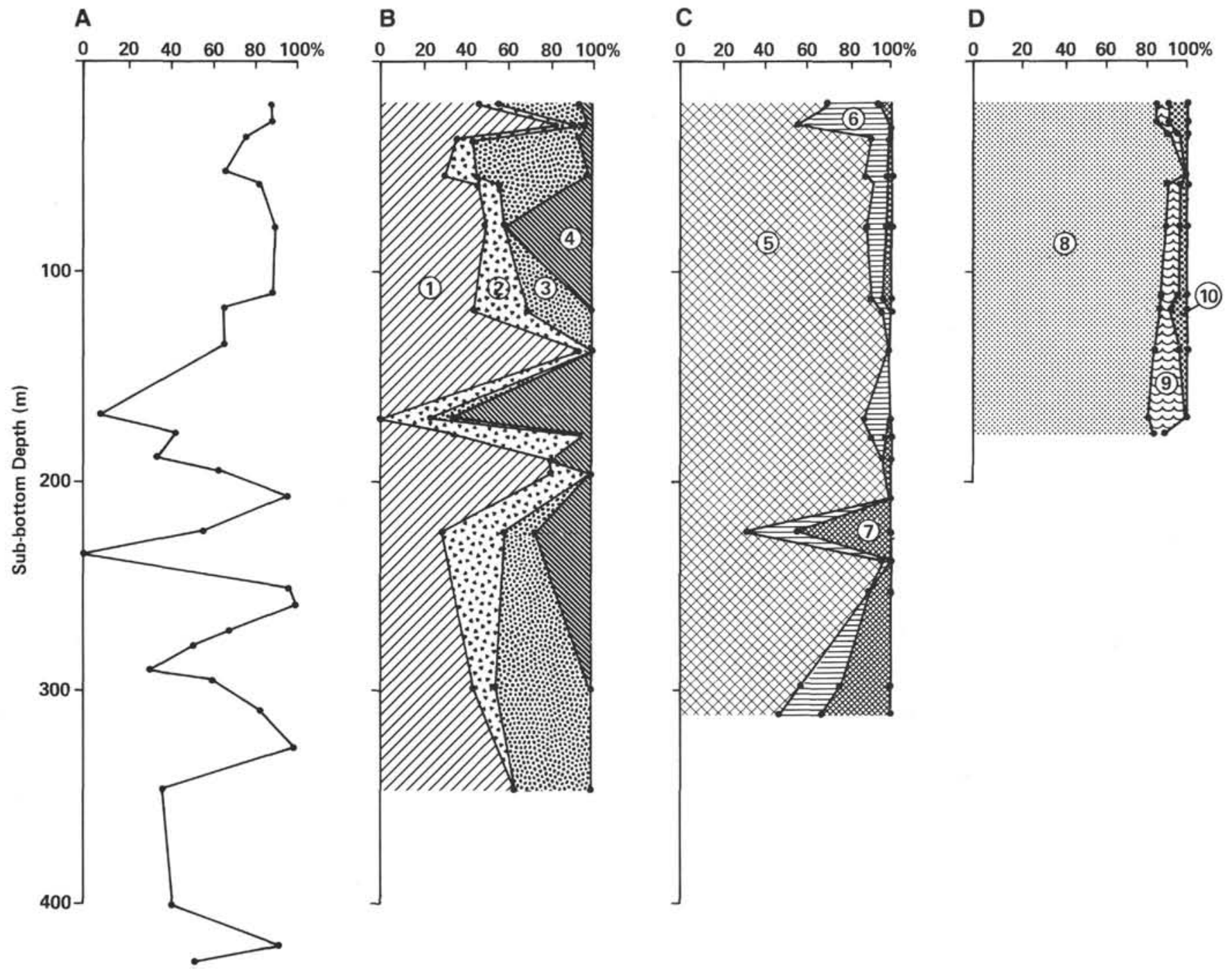

Figure 3. Changes in sediment mineralogy with sub-bottom depth for Site 495. (Symbols are identified in the Fig. 1 caption.)

\section{DISCUSSION}

According to their mineralogy, the sediments recovered during Leg 67 belong to two major types: (1) a feldspar + biogenic silica + smectite association with volcanic glass and authigenic pyrite; (2) biogenic-calcareous sediments with "metalliferous" mineralogy (ferromanganese micronodules, authigenic Fe-montmorillonite, and barite) in basal layers.

The first type predominates on the continental slope of the Middle America Trench at Sites 494 and 496, ranging in age from the Upper Cretaceous to the Holocene, and it also occurs in the upper Miocene or Pliocene to Holocene units on the Trench floor at Sites 499, 500 and in the upper units on the Cocos Plate (Site 495). The sediments are composed of terrigenous clay (mainly smectite, with minor amounts of mica, kaolinite, and chlorite), terrigenous and volcaniclastic minerals (plagioclase, volcanic glass with minor quartz, mica, and heavy minerals), and biogenic silica (diatoms with minor radiolarians and sponge spicules). Authigenic minerals, typical of hemipelagic sediments enriched in or- ganic matter and amorphous silica, are those formed in reducing conditions of early diagenesis (pyrite, glauconite, clinoptilolite ${ }^{2}$, opal C-T, diagenetic carbonate, gypsum) (Murdmaa et al., 1979; Hein et al., 1979).

The similarity of the terrigenous and volcaniclastic constituents at all sites indicates that they came from a single constant source during the whole interval from the Late Cretaceous to Recent. The mid-American landmass is a likely candidate. The assemblages of both clay and clastic minerals show that the source rocks most likely belong to circum-Pacific andesitic-belt formations (Murdmaa and Kazakova, 1980), including both altered (smectite, clinoptilolite?) and unaltered (plagioclase, pyroxenes, hornblende, colorless volcanic glass) volcaniclastic constituents. A low quartz to feldspar ratio and high pyroxene and hornblende content in heavy fraction, along with rare stable accessories and unaltered colorless volcanic glass, are indicative of an immature sediment mineralogy. Fresh volcanic ash, synchronous to sedimentation, generally pervades the section.

\footnotetext{
${ }^{2}$ Clinoptilolite may be partly of terrigenous origin.
} 
Differences in sediment mineralogy between sites and between time intervals are minor. Sediments on the upper continental slope (Site 496) and on the Trench floor (Sites 499, 500) contain more clastic feldspars and kaolinite and less smectite and chlorite than those from the lower continental slope (Site 494) and from the Cocos Plate margin (Site 495). The latter differ from all others, showing greatest smectite and lowest feldspar content, highest volcanic glass to feldspar ratio in coarse silt fraction, and most variable heavy fraction composition. These features may be explained by mechanical fractionation of the terrigenous matter during long-distance transportation and by addition of minerals from other source areas. The mineralogy of the Trench floor hemipelagic and especially turbiditic sediments is closer to that of the continental slope and differs from that of the oceanic floor.

The dominant mineralogy of nannofossil chalk (the second sediment type) at Sites 495,499 , and 500 is typical of pelagic sediments, as only calcite was found without admixture of shallow-water carbonates, such as aragonite or magnesian calcite. Siliceous biogenic skeletons, especially abundant radiolarians, indicate deposition beneath the equatorial high-productivity zone, and abundant foraminifers indicate deposition above the lysocline. Authigenic pyrite found in calcareous rocks, although partly oxidized, suggests that sedimentation took place in a reducing environment near the continental margin.

The basal manganiferous layer resembles that of midoceanic metalliferous sediments, but has undergone considerable diagenetic or hydrothermal alteration, as shown by the crystallization of authigenic smectite into ribbonlike or fibrous crystals, as well as by barite crystals and ferromanganese micronodules.

The sedimentation history of the Middle America Trench area, as indicated by mineralogy of sediments recovered during Leg 67, may be summarized as follows. Beginning in the Late Cretaceous, the sedimentation on the continental slope was continuously supplied by a smectite- and plagioclase-rich terrigenous land source area. By the middle Miocene a volcanic source existed, as indicated by the presence of volcanic glass in the coarse fraction of Core 27 from Hole 494A. After this time the volcaniclastic constituents persisted up to the Recent. During the entire interval the continental slope was an environment of high biological productivity, as indicated by the abundance of biogenic silica throughout the sediment section recovered at Sites 494 and 496.

On the Cocos Plate, sedimentation began in the early Miocene with an accumulation of metalliferous foraminifer-nannofossil ooze above basaltic basement. The basal manganiferous chalk at Sites 495,499 , and 500 is most likely a typical mid-oceanic metalliferous sediment, formed near a spreading center acting as an exhalative source of $\mathrm{Fe}$ and $\mathrm{Mn}$. Along with high $\mathrm{Fe}$ and Mn content relative to $\mathrm{Al}$ (Kurnosov, Shevchenko, this volume) we found ferromanganese micronodules and barite in the sediments. Fe-montmorillonite, of both ribbonlike and fibrous morphology, was previously found in Recent Red Sea hydrothermal sediments (Kossovskaya, 1975; Butuzova et al., 1979), but it also occurs in the Site 495,499 and 500 chalks. The exhalative-sedimentation process may have been combined with postsedimentation hydrothermal alteration of basal layers of the sediment column due to extraction of $\mathrm{Fe}, \mathrm{Mn}$, and $\mathrm{Ca}$ from underlying basalts, as shown by numerous experiments (Hajash, 1975; Mottl and Holland, 1978; Mottl et al., 1979).

The calcareous sediments at Sites 495, 499 and 500 accumulated synchronously with continental slope siliceous-terrigenous mud. Terrigenous matter did not reach the carbonate sedimentation area on the Cocos Plate either because of the long distance or topographic restrictions (like the formation of the Trench or the accumulation of carbonates on a separated ridge). The sites, however, were certainly located within an equatorial high-productivity zone.

A middle Miocene to early Pliocene hiatus at Site 499 corresponds partly to a radiolarian-bearing pelagic clay interbed at Site 495 . The supply of terrigenous sediment was weak; therefore, the site must have been far from land. During this time interval the sites subsided below the CCD as the Cocos Plate moved away from its spreading center toward the subduction zone in the Middle America Trench.

In the late Miocene, Site 495 approached the terrigenous hemipelagic sedimentation area; at this time hemipelagic mud began to accumulate over the pelagic section. A marked increase in terrigenous and volcaniclastic supply with corresponding development of hemipelagic sedimentation was noted in the late Miocene in many other regions around the Pacific (Kurnosov et al., 1980).

In the Pleistocene, hemipelagic sedimentation was substituted for turbidite accumulation at Sites 499 and 500 as these sites reached their present location on the Trench floor.

\section{ACKNOWLEDGMENTS}

The authors acknowledge Dr. N. Skornyakova and Prof. A. Lisitsyn (both of the Institute of Oceanology, U.S.S.R. Academy of Sciences) for their critical review of the manuscript.

\section{REFERENCES}

Biscaye, P. E., 1964. Mineralogy and sedimentation of the deep-sea sediment fine fraction in the Atlantic Ocean. Geochem. Techn. Rep., 8:803.

Butuzova, G. Y., Dritz, V. A., Lisitsina, N. A., Tsipursky, S. I., and Dmitrik, A. L., 1979. Dynamics of clay mineral formation in ore bearing sediments of the Atlantis-II basin. Lithol. Miner. Resour. Engl. Transl., 1:30-32.

Hajash, A., 1975. Hydrothermal process along mid-ocean ridges: an experimental investigation. Contrib. Mineral. Petrol., 53:205-226.

Hein, J. R., O'Nell, J. R., and Jones, M. G., 1979. Origin of authigenic carbonates in sediments from the deep-Bering Sea. Sedimentology, 26:681-705.

Kossovskaya, A. G., 1975. Geocrystallochemistry in the solution of geological problems. In Kossovskaya, A. G. (Ed.), Crystallochemistry of Minerals and Geological Problems: Moscow (Nauka), pp. 7-18.

Kurnosov, V., Zeitlin, N., and Narnov, G., 1980. Clay minerals: paleogeographic and diagenetic aspects. In Scientific Party, Init. Repts. $D S D P, 56,57$, Pt. 2: Washington (U.S. Govt. Printing Office), 979-1003. 
Table 5. Mineral composition of sediments, X-ray diffraction data, Sites 499 and 500.

\begin{tabular}{|c|c|c|c|c|c|c|c|c|c|c|c|c|c|}
\hline \multirow{3}{*}{$\begin{array}{c}\text { Sample } \\
\text { (core-section, } \\
\text { interval in } \mathrm{cm} \text { ) }\end{array}$} & \multirow[b]{3}{*}{$\begin{array}{l}\text { Sub-bottom } \\
\text { Depth }(\mathrm{m})\end{array}$} & \multirow[b]{3}{*}{ Unit } & \multicolumn{11}{|c|}{ Clay Minerals } \\
\hline & & & \multicolumn{3}{|c|}{ Smectite } & \multicolumn{3}{|c|}{ Mica } & \multicolumn{3}{|c|}{$\begin{array}{c}\text { Kaolinite + } \\
\text { Chlorite }\end{array}$} & \multicolumn{2}{|c|}{$\mathrm{z}^{\mathrm{b}}$} \\
\hline & & & $\begin{array}{l}(<2) \\
(\mu \mathrm{m})\end{array}$ & $\begin{array}{c}(2-20) \\
(\mu \mathrm{m})\end{array}$ & $\begin{array}{c}(>20) \\
(\mu \mathrm{m})\end{array}$ & $\begin{array}{l}(<2) \\
(\mu \mathrm{m})\end{array}$ & $\begin{array}{c}(2-20) \\
(\mu \mathrm{m})\end{array}$ & $\begin{array}{c}(>20) \\
(\mu \mathrm{m})\end{array}$ & $\begin{array}{l}(<2) \\
(\mu \mathrm{m})\end{array}$ & $\begin{array}{c}(2-20) \\
(\mu \mathrm{m})\end{array}$ & $\begin{array}{c}(>20) \\
(\mu \mathrm{m})\end{array}$ & $\begin{array}{l}(<2) \\
(\mu \mathrm{m})\end{array}$ & $\begin{array}{c}(2-20) \\
(\mu \mathrm{m})\end{array}$ \\
\hline \multicolumn{14}{|l|}{ Hole 499} \\
\hline $1-1,142-146$ & 1.42 & 1 & 80 & 88 & & 8 & & & 12 & 12 & & 0.5 & 0.6 \\
\hline $3-2,80-84$ & 12.80 & & 76 & 90 & & 20 & 4 & & 14 & 6 & & 0.5 & 0.5 \\
\hline $5-2,10-14$ & 31.10 & & 89 & & & 3 & & & 8 & & & 0.6 & \\
\hline $6-4,1-5$ & 43.51 & & 80 & & & 5 & & & 15 & & & 0.3 & \\
\hline $9-1,83-88$ & 68.33 & & 84 & 81 & & 5 & 3 & & 11 & 16 & & 0.3 & 0.4 \\
\hline $10-4,33-38$ & 81.83 & & 80 & & & 5 & & & 15 & & & 0.3 & \\
\hline $12-3,58-63$ & 99.58 & & 86 & & & 4 & & & 10 & & & 0.3 & \\
\hline $14-2,114-119$ & 111.64 & & 77 & & & 14 & & & 9 & & & 0.5 & \\
\hline $15-2,46-48$ & 126.46 & 2 & 85 & 86 & & 5 & 5 & & 10 & 9 & & 0.5 & 0.6 \\
\hline $18-4,80-84$ & 158.30 & & 93 & 89 & & 1 & 2 & & 6 & 9 & & 0.6 & 0.6 \\
\hline $22-1,20-25$ & 191.20 & & 92 & 88 & & 2 & 7 & & 6 & 5 & & 0.6 & 0.6 \\
\hline $23-1,44-49$ & 200.94 & & 92 & & & 6 & & & 2 & & & 0.4 & \\
\hline \multicolumn{14}{|l|}{ Hole 499B } \\
\hline $4-1,59-64$ & 230.05 & 4 & $\mathrm{tr}$ & $\operatorname{tr}$ & & - & - & & - & - & & & \\
\hline $6-1,72-77$ & 249.27 & & tr & & & - & & & - & & & & \\
\hline $8-3,10-14$ & 270.60 & & $\operatorname{tr}$ & & & - & & & - & & & & \\
\hline $9, \mathrm{CC}(25-27)$ & 278.75 & 5 & tr & $\mathrm{tr}$ & $\operatorname{tr}$ & $\operatorname{tr}$ & - & - & - & - & - & & \\
\hline \multicolumn{14}{|l|}{ Hole 500} \\
\hline $15-5,85-89$ & 134.85 & 3 & $\operatorname{tr}$ & & & $\operatorname{tr}$ & & & - & & & & \\
\hline $17-1,123-127$ & 148.23 & 4 & tr & $\operatorname{tr}$ & tr & - & - & - & - & - & - & & \\
\hline $17, \mathrm{CC}(18-22)$ & 156.18 & & $\mathrm{tr}$ & & & - & & & - & & & & \\
\hline
\end{tabular}

Note: $++=$ abundant $+=$ moderate; $\mathrm{tr}=$ trace; $-=$ not found.

a Samples tested with $10 \% \mathrm{HCl} 1$ hour at $90^{\circ}-100^{\circ} \mathrm{C}$.

b Coefficient $\mathrm{Z}$ indicates the ratio of $17 \AA$-peak shoulders (see the text for an explanation).

Mottl, M. J., and Holland, H. D., 1978. Chemical exchange during hydrothermal alteration of basalt by seawater. Geochim. Cosmochim. Acta, 42:1103-1115.

Mottl, M. J., Holland, H. D., and Corr, R. F., 1979. Chemical exchange during hydrothermal alteration of basalt by seawater. II. Experimental results for $\mathrm{Fe}, \mathrm{Mn}$, and sulfur species. Geochim. Cosmochim. Acta, 43:869-884.
Murdmaa, I. O., and Kazakova, V., 1980. Coarse silt fraction mineralogy of the Japan Trench sediments. In Scientific Party, Init. Repts. DSDP, 56, 57, Pt. 2: Washington (U.S. Govt. Printing Office), $1005-1009$.

Murdmaa, I. O., Serova, V. V., Lisitsin, A. P., and Emelyanov, E. M., 1979. Clastic terrigenous and volcanogenic minerals of sand-silt fraction. In Bezrukov, P. L. (Ed.), Sedimentation and Magmatism in Oceans. Oceanic Geology: Moscow (Nauka), pp. 180-197. 
Table 5. (Continued).

\begin{tabular}{|c|c|c|c|c|c|c|c|c|c|c|c|c|c|c|c|}
\hline \multirow{3}{*}{$\begin{array}{c}\begin{array}{c}\text { Clay } \\
\text { Minerals }\end{array} \\
\mathrm{Z}^{\mathrm{b}} \\
\begin{array}{c}(>20) \\
(\mu \mathrm{m})\end{array}\end{array}$} & \multicolumn{15}{|c|}{ Other Minerals } \\
\hline & \multicolumn{3}{|c|}{ Quartz } & \multicolumn{3}{|c|}{ Feldspar } & \multicolumn{3}{|c|}{ Clinoptilolite } & \multicolumn{3}{|c|}{ Calcite } & \multicolumn{3}{|c|}{ Amorphous Phase } \\
\hline & $\begin{array}{l}(<2) \\
(\mu \mathrm{m})\end{array}$ & $\begin{array}{c}(2-20) \\
(\mu \mathrm{m})\end{array}$ & $\begin{array}{c}(>20) \\
(\mu \mathrm{m})\end{array}$ & $\begin{array}{l}(<2) \\
(\mu \mathrm{m})\end{array}$ & $\begin{array}{c}(2-20) \\
(\mu \mathrm{m})\end{array}$ & $\begin{array}{c}(>20) \\
(\mu \mathrm{m})\end{array}$ & $\begin{array}{l}(<2) \\
(\mu \mathrm{m})\end{array}$ & $\begin{array}{c}(2-20) \\
(\mu \mathrm{m})\end{array}$ & $\begin{array}{c}(>20) \\
(\mu \mathrm{m})\end{array}$ & $\begin{array}{l}(<2) \\
(\mu \mathrm{m})\end{array}$ & $\begin{array}{c}(2-20) \\
(\mu \mathrm{m})\end{array}$ & $\begin{array}{c}(>20) \\
(\mu \mathrm{m})\end{array}$ & $\begin{array}{l}(<2) \\
(\mu \mathrm{m})\end{array}$ & $\begin{array}{c}(2-20) \\
(\mu \mathrm{m})\end{array}$ & $\underset{(\mu \mathrm{m})}{(>20)}$ \\
\hline & tr & + & & + & ++ & & - & tr & & - & - & & tr & tr & \\
\hline & $\operatorname{tr}$ & tr & & + & + & & $\operatorname{tr}$ & $\operatorname{tr}$ & & - & - & & tr & tr & \\
\hline & tr & & & tr & & & tr & & & - & & & tr & & \\
\hline & tr & & & + & & & - & & & - & & & $\operatorname{tr}$ & & \\
\hline & tr & $\operatorname{tr}$ & & + & + & & $\operatorname{tr}$ & tr & & - & - & & tr & tr & \\
\hline & tr & & & + & & & - & & & - & & & tr & & \\
\hline & tr & & & $\begin{array}{lll}+ & & l\end{array}$ & & & $\operatorname{tr}$ & & & - & & & tr & & \\
\hline & tr & & & + & & & - & & & - & & & $\operatorname{tr}$ & & \\
\hline & tr & + & & + & ++ & & tr & tr & & - & - & & $\operatorname{tr}$ & tr & \\
\hline & tr & + & & $\operatorname{tr}$ & + & & tr & tr & & - & - & & tr & tr & \\
\hline & tr & $\operatorname{tr}$ & & + & $\operatorname{tr}$ & & tr & $\operatorname{tr}$ & & - & - & & $\operatorname{tr}$ & tr & \\
\hline & tr & & & tr & & & tr & & & - & & & tr & & \\
\hline & - & - & & - & - & & - & - & & ++ & ++ & & - & - & \\
\hline & - & & & - & & & - & & & ++ & & & - & & \\
\hline & $\overline{-}$ & - & - & $\overline{-}$ & - & - & - & - & - & ++ & ++ & ++ & - & - & - \\
\hline & - & & & - & & & - & & & ++ & & & - & & \\
\hline & $\overline{-}$ & - & - & $\bar{z}$ & - & - & - & - & - & ++ & ++ & ++ & - & - & - \\
\hline
\end{tabular}
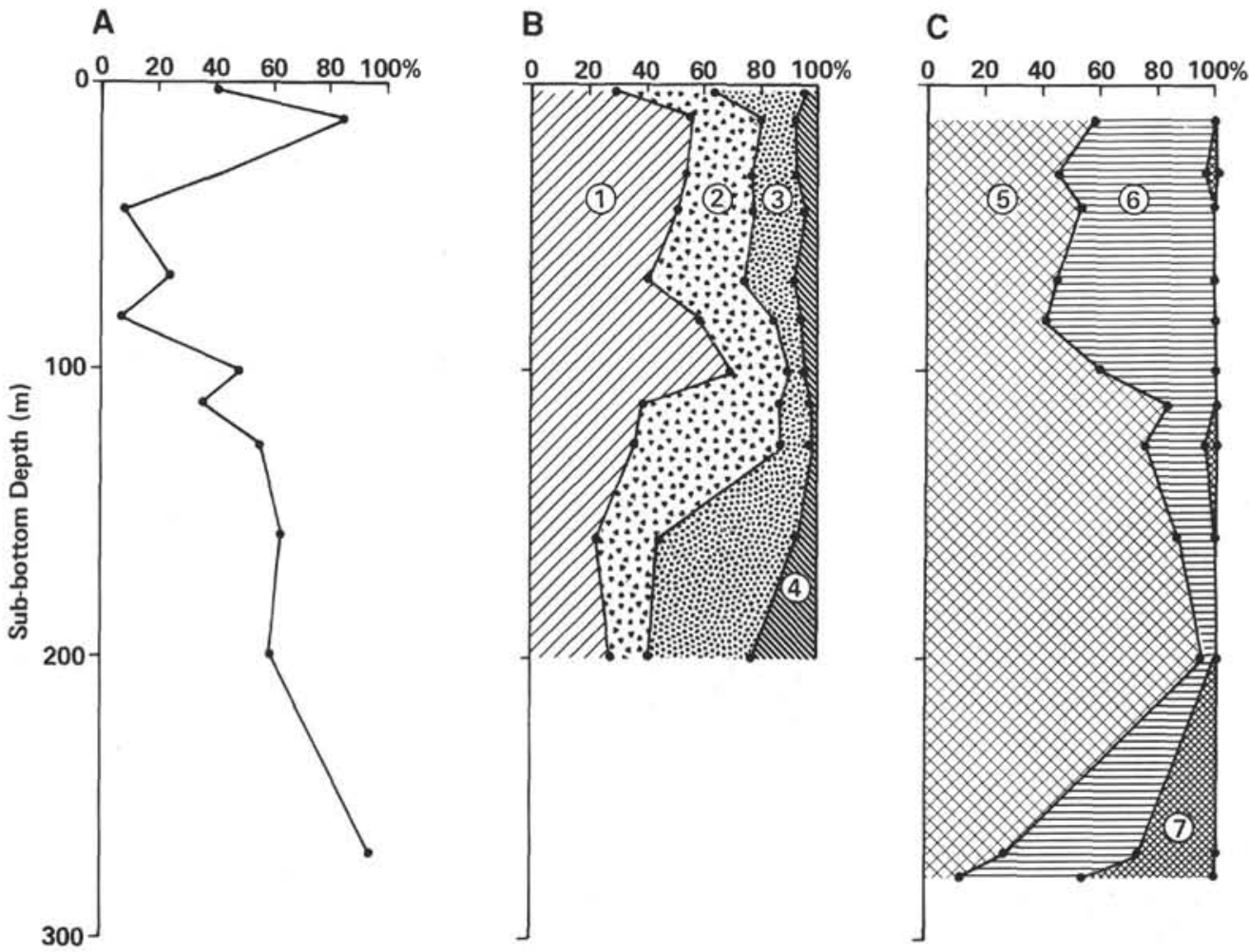

D

Figure 4. Changes in mineralogy of sediments with sub-bottom depth for Site 499. (The caption for Fig. 1 explains the symbols.) 


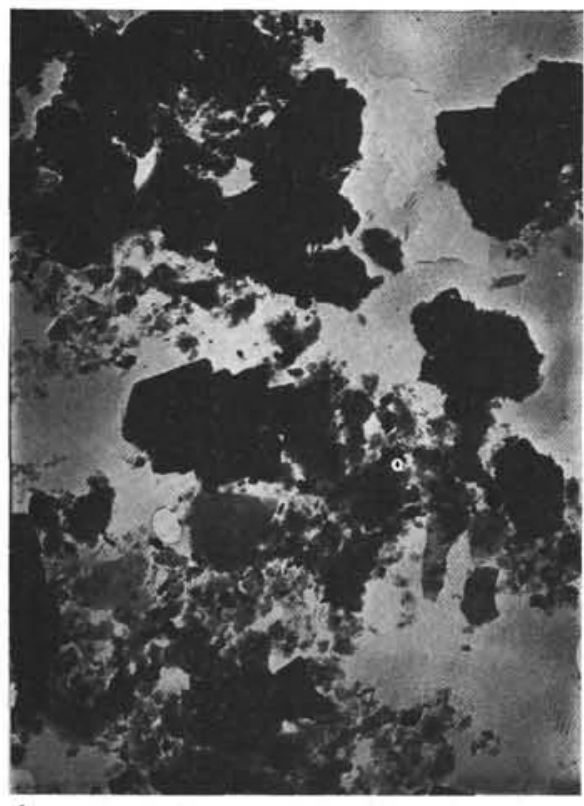

1

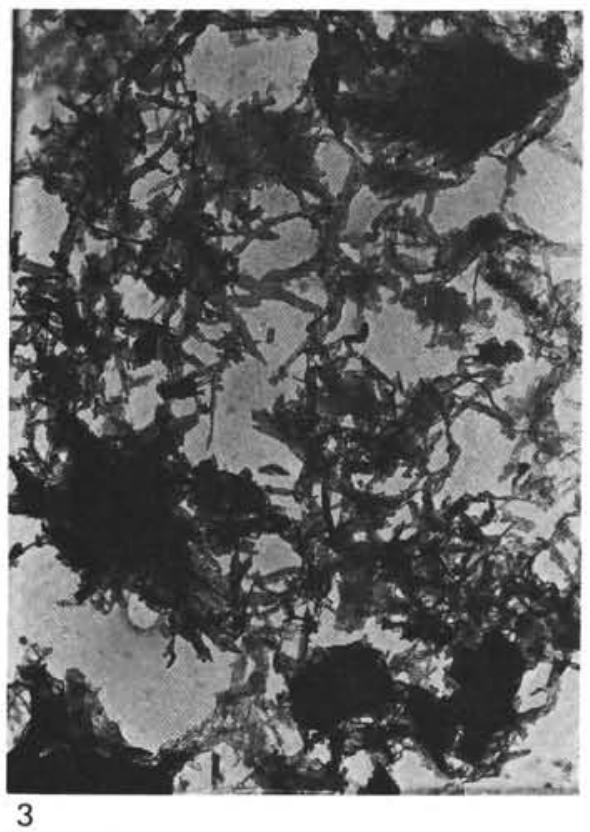

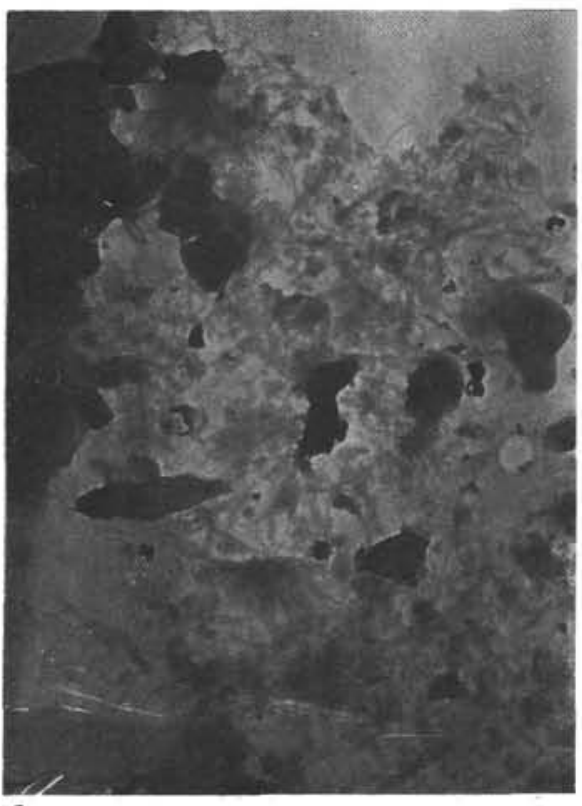

2

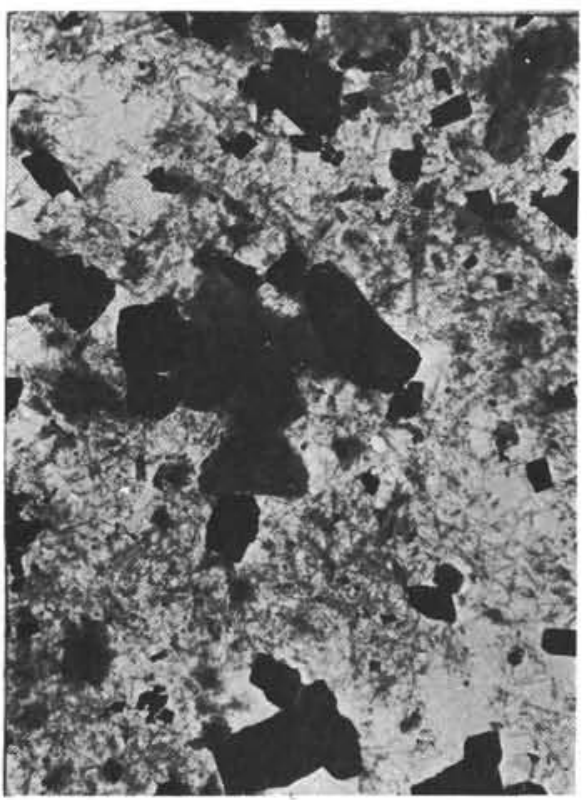

4

Plate 1. Electron microscope photographs of clay minerals of sediments from the Middle America Trench, Leg $67(<2 \mu \mathrm{m})$. 1 . Smectite (irregular flakes) and kaolinite (angular hexagonal plate). Sample 496-3-1, 50-54 cm $(\times 12,000) .2-4$. Authigenic smectites from carbonate sediments, (2) Sample 495-39-3, 50-52 cm ( $\times 12,000)$, (3) Sample 499B-9, CC 25-27 cm ( $\times 12,000)$, (4) Sample 500-17-1, 123-127 cm ( $\times 10,000)$. 